\title{
Membranous Nephropathy and Anti-Podocytes Antibodies: Implications for the Diagnostic Workup and Disease Management
}

\author{
Agnieszka Pozdzik (D), ${ }^{1,2}$ Isabelle Brochériou, ${ }^{3,4}$ Cristina David, ${ }^{1}$ Fahd Touzani, ${ }^{1}$ \\ Jean Michel Goujon, ${ }^{5,6}$ and Karl Martin Wissing ${ }^{1,7}$ \\ ${ }^{1}$ Nephrology Department, Centre Hospitalier Universitaire de Bruxelles (CHUB), Brugmann Hospital, Brussels, Belgium \\ ${ }^{2}$ Université Libre de Bruxelles (ULB), Brussels, Belgium \\ ${ }^{3}$ Pathology Department, AP-HP, Pitié Hospital, Paris, France \\ ${ }^{4}$ UPMC Université Paris 6, Paris, France \\ ${ }^{5}$ Centre National de Référence Maladies Rares: Amylose AL et Autres Maladies à Dépôts d'Immunoglobulines Monoclonales, \\ Université de Poitiers, Poitiers, France \\ ${ }^{6}$ Pathology Department, Centre Hospitalier Universitaire de Poitiers, Poitiers, France \\ ${ }^{7}$ Nephrology Department, Universitair Ziekenhuis Brussel, Vrije Universiteit Brussel, Brussels, Belgium
}

Correspondence should be addressed to Agnieszka Pozdzik; agnieszka.pozdzik@chu-brugmann.be

Received 11 June 2017; Revised 31 August 2017; Accepted 15 October 2017; Published 8 January 2018

Academic Editor: Michael Mahler

Copyright (C) 2018 Agnieszka Pozdzik et al. This is an open access article distributed under the Creative Commons Attribution License, which permits unrestricted use, distribution, and reproduction in any medium, provided the original work is properly cited.

The discovery of circulating antibodies specific for native podocyte antigens has transformed the diagnostic workup and greatly improved management of idiopathic membranous nephropathy (iMN). In addition, their identification has clearly characterized iMN as a largely autoimmune disorder. Anti-PLA2R1 antibodies are detected in approximately $70 \%$ to $80 \%$ and anti-THSD7A antibodies in only $2 \%$ of adult patients with iMN. The presence of anti-THSD7A antibodies is associated with increased risk of malignancy. The assessment of PLA2R1 and THSD7A antigen expression in glomerular immune deposits has a better sensitivity than measurement of the corresponding autoantibodies. Therefore, in the presence of circulating anti-podocytes autoantibodies and/or enhanced expression of PLA2R1 and THSD7A antigens MN should be considered as primary MN (pMN). Anti-PLA2R1 or anti-THSD7A autoantibodies have been proposed as biomarkers of autoimmune disease activity and their blood levels should be regularly monitored in pMN to evaluate disease activity and predict outcomes. We propose a revised clinical workup flow for patients with MN that recommends assessment of kidney biopsy for PLA2R1 and THSD7A antigen expression, screening for circulating anti-podocytes antibodies, and assessment for secondary causes, especially cancer, in patients with THSD7A antibodies. Persistence of anti-podocyte antibodies for 6 months or their increase in association with nephrotic proteinuria should lead to the introduction of immunosuppressive therapies. Recent data have reported the efficacy and safety of new specific therapies targeting B cells (anti-CD20 antibodies, inhibitors of proteasome) in pMN which should lead to an update of currently outdated treatment guidelines.

\section{Introduction}

After a short review of the pathophysiology of membranous nephropathy $(\mathrm{MN})$, in the first part of this overview article, we describe the recently identified nephrotoxic antibodies and the corresponding antigens expressed by podocytes.
They mainly consist of antibodies directed against neutral endopeptidase (NEP) in newborns [1], the cationic bovine serum albumin (BSA) [2] and aryl sulfatase in childhood [3], as well as the M-type phospholipase A2 receptor type 1 (PLA2R1) [4] and thrombospondin type 1 domain containing 7A (THSD7A) [5] in adults. 
Antibodies against PLA2R1 are of particular clinical importance as they are detected in approximately $70 \%$ to $80 \%$ of adult cases of MN without apparent secondary causes, particularly in men $[4,6]$. The prevalence of anti-PLA2R1 related $\mathrm{MN}$ appears to be lower (about 53\%) in Japan than in other countries $[7,8]$. Anti-THSD7A antibodies are detected in only $2 \%$ of adult patients with iMN with higher prevalence in women $[5,7]$. The prevalence of anti-THSD7A related $\mathrm{MN}$ was $5.5 \%$ (7 out of 117 PLA2R1 negative MN cases) according to the immunoperoxidase staining detecting granular THSD7A antigen expression within extramembranous deposits [9] and 6.1\% (4 out of 66) in a European cohort of PLA2R1 negative MN cases naïve of any immune therapy [5]. Antibodies against both PLA2R1 and THSD7A have been reported in about $1 \%$ of $\mathrm{MN}$ [9].

Both autoantibodies have been proposed as biomarkers of $\mathrm{MN}$ autoimmune activity [10-12]. High anti-PLA2R1 antibody levels have recently been reported as a reliable prognostic factor [13-16] which is likely to modify the indications for treatment to improve long-term outcomes of $\mathrm{MN}$ in the future.

More intensive screening for malignancy has been proposed in patients with THSD7A-related MN based on data from a cohort of 1276 patients with MN. Among these 8 women out of 40 with THSD7A-related MN developed cancer within 3 months from the diagnosis of $\mathrm{MN}$ [17].

\section{Clinical Characteristics of Membranous Nephropathy}

Membranous nephropathy (MN) is the most common cause of nephrotic syndrome in adults ( $\approx 25 \%$ of cases). The prevalence of $\mathrm{MN}$ varies widely by geographic regions and attains "epidemic" levels in China [10,18]. Men are two times more likely to be affected by this disease than women [19]. MN occurs at any age, although it is rarely observed in children $(10 \%)[20,21]$. The incidence of $\mathrm{MN}$ increases progressively with age, with a peak between 30 and 50 years [15, 19, 22-25].

$\mathrm{MN}$ is the $2 \mathrm{nd}$ or $3 \mathrm{rd}$ most common form of primary glomerulonephritis and evolves to end-stage renal diseases (ESRD) in $30 \%$ of patients [18]. In most cases, the onset of $\mathrm{MN}$ is not preceded by any prodromal manifestations such as signs of infection. Most patients present with a nephrotic syndrome: proteinuria above $3.0 \mathrm{~g} / 24 \mathrm{~h}$, hypoalbuminemia, edema, hyperlipidemia and lipiduria, and normal, or slightly altered, kidney function. The incidence of $\mathrm{MN}$ is probably underestimated given that the proteinuria below $2.0 \mathrm{~g} / \mathrm{day}$ without a nephrotic syndrome has been described in $10 \%$ to $20 \%$ of cases [10]. Arterial hypertension has been reported in $10 \%$ to $55 \%$ of case series and is associated with a progressive decline of renal function $[26,27]$. Acute renal vein thrombosis associated or not with pulmonary embolism has been rarely reported as the initial presentation of $\mathrm{MN}[28,29]$.

Spontaneous complete remission of proteinuria is observed after a variable period of time (4 to 120 months) in approximately $30 \%$ to $40 \%$ of adult patients [30, 31]. In face of a sudden deterioration of the kidney function, tests should focus on the possibility of focal or diffuse lupus nephritis [32]. Primary MN may also be complicated by the development of focal and segmental glomerulosclerosis or by crescentic glomerulonephritis due to the development of anti-neutrophil cytoplasmic antibodies or antiglomerular basement antibodies [33].

\section{The Clinical Forms of $\mathbf{M N}$}

Two forms of MN have classically been described: the idiopathic form and the secondary form of $\mathrm{MN}$ which represent $70 \%$ and $30 \%$ of cases, respectively $[11,12,34]$.

Secondary $\mathrm{MN}$ is associated with the presence of immune complexes possibly containing foreign antigens and is most often observed in children as well as in patients over the age of 60 . It occurs in the context of a number of infections including viral hepatitis $\mathrm{B}(\mathrm{HBV})$, hepatitis $\mathrm{C}$, and hepatitis E or syphilis [35-37], certain autoimmune diseases (systemic lupus erythematosus, antineutrophil, Hashimoto's thyroiditis, and Sjögren's syndrome) [32, 33, 38], hyper-IgG4 syndrome [39], malignancies (digestive tract, lungs, and breast) [40], and the use of medicinal products (heavy metal salts such as gold salts in particular, D-penicillamine and its derivatives, nonsteroidal anti-inflammatory drugs, and levamisoleadulterated cocaine [41]) [41-44]. It has also been described in association with diseases such as sarcoidosis, in both adults [45-49] and children [50], sickle-cell anaemia, and graftversus-host disease $[51,52]$.

Primary $\mathrm{MN}$ is related to the autoimmune disorders caused by the presence of circulating antibodies against native podocyte antigens NEP [1], PLA2R1 [4], and THSD7A [5] or by antibodies developed against external antigens such as cationic bovine serum albumin (BSA) [2] or aryl sulfatase [3]. Screening for these pathogenic antibodies therefore provides a new opportunity to more accurately define the etiology and the underlying pathways in the development of MN. The MN has been reported in Fabry disease [53]; however as far as we know the presence of anti- $\alpha$-galactosidase A antibodies has not been documented.

\section{Histological Features in $\mathrm{MN}$}

The key histological characteristic of $\mathrm{MN}$ is the formation of subepithelial (extramembranous) immune deposits, associated with variable degrees of alterations in the morphology of the glomerular basement membrane ("spikes"). The histology of $\mathrm{MN}$ depends on the timing of renal biopsy with respect to the onset of the disease and reflects the stage of disease [18]. Glomerular abnormalities, which are always diffuse, can be classified into 4 stages (Figure 1). Immunofluorescence is a useful technique in the diagnostic workup because it reveals the type and subtypes of immunoglobulin $G$ within the deposits. Recent data suggest that the type of IgG subclasses in deposits provides important information on disease mechanisms and the potential for complement activation $[54,55]$. All IgG subclasses have been observed in immune deposits. IgG1, IgG2, and IgG3 deposits are reported more frequently in secondary MN (lupus [56]; graft-versus host disease [57]; malignancies [58]). The IgG4 subclass has been 


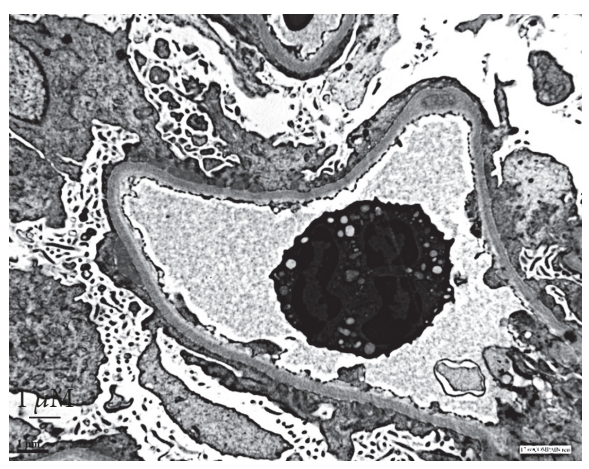

(a) MN Stage I

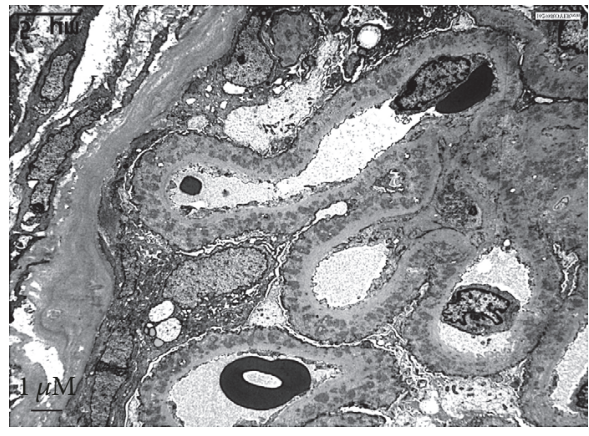

(c) MN Stage III

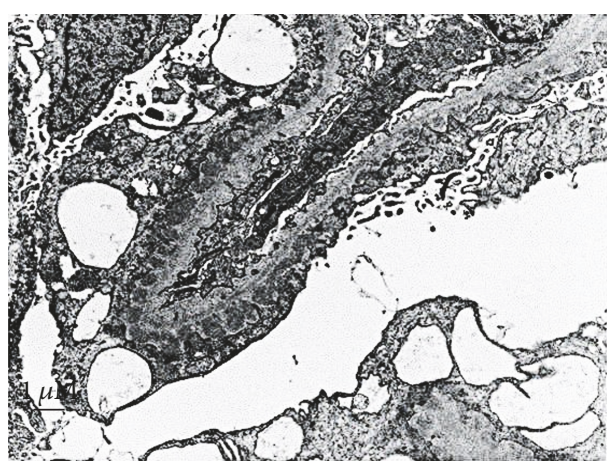

(b) MN Stage II

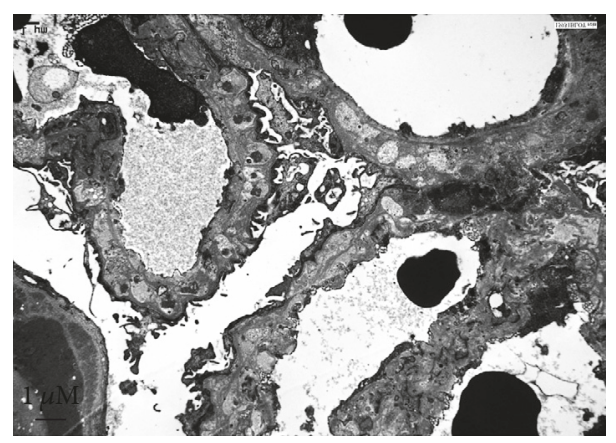

(d) MN Stage IV

FIGURE 1: Electron microscopy representative image of glomerular membranous nephropathy in adults. (a) Stage I: electron dense deposits, irregularly distributed, at the outside of the glomerular basement membrane (GBM), without inflammatory reaction around the deposits. There is a variable degree of foot process effacement. (b) Stage II: spikes are irregular projections of the GBM among the subepithelial deposits. (c) Stage III: with progression of the disease, spikes become longer and incorporate the deposits in a thickened GBM. (d) Stage IV: the deposits lose their electron density until disappearance in the advanced stages of the process. Magnification $\times 3000$. Kindly provided by Jean Michel Goujon, M.D., Ph.D. (Centre National de Référence Maladies Rares: Amylose AL et Autres Maladies à Dépôts d'Immunoglobulines Monoclonales, Université de Poitiers; Pathology Department, Centre Hospitalier Universitaire de Poitiers, Poitiers, France).

most often observed in pMN and is in general absent in MN related to the malignancy $[24,58-60]$.

In a large cohort from Japan, specificity and sensitivity of IgG4 positive staining for detecting pMN corresponded to approximately $95 \%$ and $64 \%$, respectively [7]. Glomerular IgG4 staining has been most frequently associated with PLA2R1 deposition in pMN and is rarely observed in HBVrelated $\mathrm{MN}$ and in general absent in the membranous form of lupus nephritis [61]. The absence of glomerular IgG4 staining has been reported to be significantly more frequent in $\mathrm{MN}$ associated with malignancy than in $\mathrm{pMN}$ and has been identified as an independent predictor for occurrence of malignancy during the follow-up [59]. However, the data in the literature concerning the diagnostic value of IgG4 staining to differentiate between secondary $\mathrm{MN}$ due to malignancies and $\mathrm{pMN}$ is not completely clear-cut. In pMN related to PLA2R1 antibodies, IgG4 within extramembranous deposits is usually polytypic (involving several antibody specificities). On the contrary, monotypic IgG4 deposits have been reported in secondary forms of MN without anti-PLA2R1 antibodies in patients with hematological disorders [62] and lung neoplasia [63]. More intriguingly, the glomerular PLA2R1 and predominant/codominant IgG4 coexpression has also been reported in $\mathrm{MN}$ associated with malignancy
[61]. These reports are therefore in contradiction with the initial observation that IgG4 and anti-PLAR1 antibodies are very rare or absent in patients with malignancy [59].

The presence of more than 8 inflammatory cells per glomerulus has been proposed to increase the likelihood of secondary MN related to the cancer, which should be thoroughly excluded by extensive investigation [64]. However, the sensitivity of this marker appears to be low because secondary forms of MN due to cancer have been reported without the presence of glomerular inflammatory cells [65].

\section{Pathogenesis of Histological Lesions in MN: Glomerular Deposition of Immune Complexes, Complement Activation, and Tubulointerstitial Damage}

As in other nephropathies, the persistence of high-grade albuminuria and/or proteinuria is associated with a progressive decline of glomerular filtration rate secondary to tubulointerstitial lesions (including tubular atrophy, interstitial inflammation, and fibrosis) in $\mathrm{MN}[66,67]$. While high-grade proteinuria is unequivocally a risk factor for the progression of renal dysfunction in general, in $\mathrm{MN}$ the 
situation might be more complex. The oxidative stress related to podocyte injury and glomerular sclerosis and the use of nephrotoxic drugs such as calcineurin inhibitors and renal vein thrombosis are among other potential mechanisms that might lead to the progressive decline of renal function [11]. An increase in levels of PLA2R1 antibodies has been reported as adverse risk factor for progression of renal failure especially in older patients. This risk was 2.8 times higher in men who presented progressive increase in total anti-PLA2R1 IgG levels as compared with women [68].

In addition, growing evidence demonstrates the role of complement activation in the progression of $\mathrm{MN}$ [69]. Immune complex deposits (Figure 1) in the space between the glomerular capillary basement membranes and podocytes contain (1) antigens which are either "foreign" or intrinsic to podocytes, (2) immunoglobulin subclasses directed against those antigens, and (3) components of the complement system, including in particular the membrane attack complex (MAC) C5b-9 whose effect on the podocyte cell membrane is responsible for cell injury and rearrangements of the glomerular basement membrane (GBM) which lead to proteinuria [11, 24, 70-73].

Complement activation probably also contributes to the pathogenesis and progression of renal insufficiency in patients with $\mathrm{MN}[71,74,75]$. Indeed C5b-9 blocks podocyte autophagy by inhibition of lysosomal degradation of autophagosomes which increases podocyte apoptosis in human MN and cultured podocytes [76]. Inhibition of the podocyte autophagosomal/lysosomal system and ubiquitin proteasome system induces podocyte injury and worse proteinuria [77-79].

Moreover, tubulointerstitial injury (tubular atrophy and interstitial fibrosis) could be related to the exposure of tubular epithelial cells to various levels of protein in the tubular lumen and in particular ultrafiltrate-derived serum proteins containing complement factors (complementuria) [80]. The proximal tubular epithelial cells (PTEC) are of particular importance in the activation of urinary complement components. Indeed, the brush border on the apical membrane of PTEC bind properdin, which activates complement by the alternative pathway $[81,82]$. It has been reported that, following the cleavage of $\mathrm{C} 3$, properdin may bind to serum $\mathrm{C} 3 \mathrm{~b}$, stabilizes $\mathrm{C} 3 \mathrm{bBb}$ convertase, and thus amplifies local activation of C3 and cell lesions [83]. The expression of properdin by PTEC has been reported in proteinuric diseases [82]. An in vitro study has demonstrated that exposure of PTEC to complement results in local C3-deposition and formation of MAC (C5b9) [84]. Therefore, PTEC could hypothetically be involved in the progression of $\mathrm{MN}$ by focal activation of the alternative complement pathway leading to progressive and sustained injury of PTEC.

\section{The Podocyte Antigens and Pathogenic Antibodies}

The hypothesis of an autoimmune disease attacking podocytes was generated following observations in Heymann nephritis, an experimental model of $\mathrm{MN}$ in the rat (Figure 2(a)) [85]. In this model, megalin present on the surface of podocytes of rats has been identified as the target antigen responsible for the formation of extramembranous deposits.

Proof of the hypothesis that $\mathrm{MN}$ in humans also involves a podocyte antigen has been recently provided by two major discoveries (Figures 2(b)-2(d)). The first is the identification of neutral endopeptidase (NEP), an antigen involved in rare cases of neonatal MN [1]; the second is the characterization of antibodies directed against the PLA2R1 [4] and, more recently, against the THSD7A antigen [5].

The discovery of those autoantibodies targeting podocyte antigens has provided irrefutable proof that about $80 \%$ of $\mathrm{MN}$ previously considered as "idiopathic" are in fact caused by antibody-mediated autoimmune disease [4, 5, 86]. Accordingly the name "PLA2R1 or THSD7A related MN" needs to be used instead of iMN, which is no longer an appropriate terminology in the presence of corresponding autoantibodies [10-12]. The term iMN should therefore be restricted to the small minority of cases without specific autoantibodies and without an identifiable cause for secondary MN.

The diagnostic approach in pediatric forms of $\mathrm{MN}$ differs from adults and also requires the evaluation for (1) anti-NEP antibodies secondary to fetomaternal alloimmunization in newborns with MN and (2) deposition of specific antibodies against external antigens planted on podocytes such as cationic BSA in early childhood MN and aryl sulfatase in the case of $\mathrm{MN}$ in patients receiving enzyme substitution therapy [1-3].

6.1. Neutral Endopeptidase (NEP) and Fetomaternal Alloimmunization Related MN. Pierre Ronco and his team first identified a podocyte antigen responsible for $\mathrm{MN}$ in humans [1]. In this exceptional case of a neonate, MN was induced by the placental transfer of maternal antibodies directed against NEP in the foetal glomeruli during the last trimester of pregnancy [1]. NEP, also called CD10 or common acute lymphoblastic leukemia antigen (CALLA), is a metalloprotease involved in the degradation of peptides (enkephalin, natriuretic factors, endothelin, etc.) [87]. It is expressed by podocytes, the brush border of proximal tubular cells, as well as by endothelial cells, granulocytes, and syncytiotrophoblasts [88]. Mothers who are carriers of a homozygous or composite heterozygous mutation of the $M M E$ gene coding for NEP do not express the protein, which is no longer detected in the urine $[89,90]$. Though the mothers have a deficiency of NEP, they are asymptomatic. During pregnancy, the immune system of these mothers is exposed for the first time to the NEP present on syncytiotrophoblasts. This leads to a fetomaternal alloimmunization process comparable to that associated with Rhesus incompatibility [91]. Anti-NEP alloantibodies of the IgG1 and IgG4 subclasses are detected in the maternal serum and breast milk, as well as transiently in the neonatal serum $[89,92]$. The antigen has also been documented in the neonatal extramembranous glomerular deposits [89]. The responsibility of maternal anti-NEP antibodies in the pathophysiology of $\mathrm{MN}$ has been confirmed in vivo via the injection of maternal antibodies [93] into rabbits, which led to the development of nephrotic proteinuria and the formation of extramembranous deposits, characteristic of $\mathrm{MN}$ [1]. This work provided the first irrefutable proof 
Megalin: first podocytes antigen in experimental model of MN
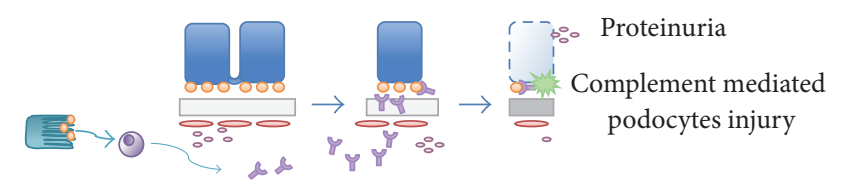

Podocyte

Megalin

- Proximal tubular cells

- Glomerular basement membrane

(a) Experimental membranous nephropathy Heymann nephritis

Phospholipase A2 receptor type 1: main podocytes antigen
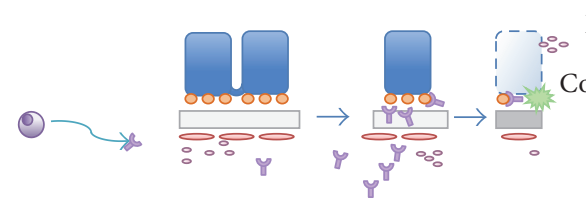

Proteinuria

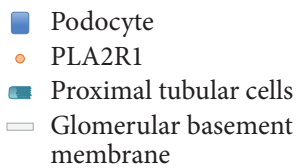

$$
\begin{aligned}
\text { - Endothelium } \\
\text { - B cells } \\
=\text { Antibody } \\
=\text { Membrane attack } \\
\text { complex (C5b9) }
\end{aligned}
$$

(c) Primary membranous nephropathy
Neutral endopeptidase: first podocytes antigen in human MN

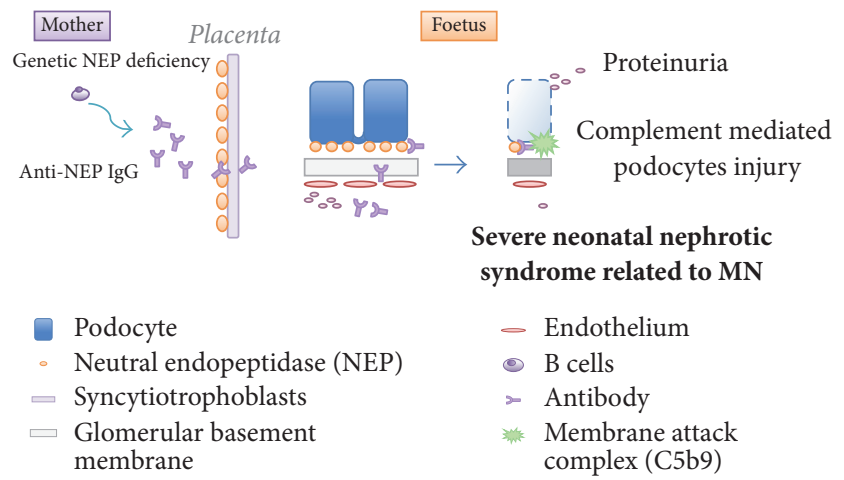

(b) Fetomaternal alloimmunization with antenatal glomerulopathies (FMAIG)

Thrombospondin type $\mathbf{1}$ domain containing 7A: rare podocytes

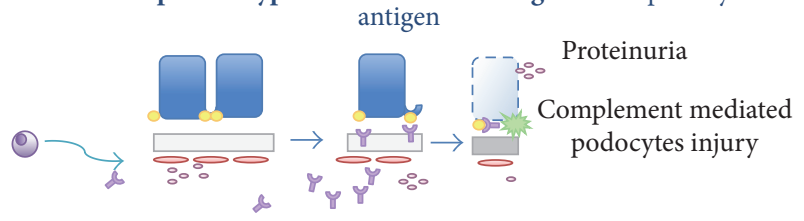

$$
\begin{aligned}
& \text { Podocyte } \quad \text { Endothelium } \\
& \text { THSD7A4 O B cells } \\
& \text { - Proximal tubular cells } \quad \text { Antibody } \\
& \square \text { Glomerular basement 洋 Membrane attack } \\
& \text { membrane complex (C5b9) }
\end{aligned}
$$

(d) Primary membranous nephropathy

FIGURE 2: Proposed mechanisms of experimental membranous nephropathy in rats (indirect alloimmunization) and human primary membranous nephropathies.

of the involvement of a podocyte antigen serving as the target for pathogenic autoantibodies, thus confirming the pathophysiological mechanisms of experimental Heymann nephritis in humans [73]. Until today, 5 additional families have been identified with the previously reported $M M E$ gene truncating mutation [90].

6.2. Anti-Phospholipase A2 Receptor Type 1 Autoantibodies. The new pathophysiological concept of alloimmune $\mathrm{MN}$ has stimulated research aiming to identify human podocyte antigens that may act as targets for circulating pathogenic antibodies in humans, thus leading the way to the identification in 2009 of the first antigen responsible for primary MN in adults [4]. This publication was a cornerstone in the history of $\mathrm{MN}$ as it provided evidence that iMN in adults is actually an autoimmune disease associated with the production of anti-PLA2R1 antibodies and introduced a new terminology of PLA2R1 related MN $[15,23,94-96]$.

Until now, the direct proof that human anti-PLA2R1 antibodies induce $\mathrm{MN}$ in experimental animal models has not been reported. Podocytes of neither rats nor mice express the PLA2R1 antigen. However, primary cultured podocytes derived from canine kidneys express PLA2R1 in vitro, which offers new promises to demonstrate the direct pathogenicity of PLA2R1 antibodies in the future [97]. The induction of $\mathrm{MN}$ by monoclonal IgG3 kappa in recurrent $\mathrm{MN}$ is a potent argument for the direct pathogenic role of these antibodies in this disease [98].

6.3. Anti-PLA2R1 Antibodies, Genetic Risk Factors for Disease, and Immune Dominant Epitopes of PLA2R1. The involvement of genetic factors, in particular increased frequency of the HLA-DRw3, HLA-B8, and B18 alleles of the HLA histocompatibility system in patients with idiopathic MN, has been published as early as 1979 [99, 100]. A genomewide association study (GWAS) investigating over 280,000 individual polymorphic markers ("single nucleotide polymorphism" or SNP) found a highly significant association between MN and certain HLA-DQA1 polymorphisms [101]. The SNPs rs3749119, rs3749117, and rs4664308 in PLA2R1 and rs2187668 in HLA-DQA1 have been significantly associated with primary MN and anti-PLA2R1 antibodies [94]. Several reports have observed associations between PLA2R1 polymorphisms and the risk for development of primary MN [102], the response to immunosuppression (in association with HLADQA1 polymorphisms) [103], response to immunosuppressive therapy (a lower rate of remission for the $\mathrm{C} / \mathrm{G}$ genotype at rs35771982) [104], and disease progression (C/T genotype at rs6757188) [104]. However, these observations remain to be independently confirmed [105].

Recently 2 independent groups have identified the region within the extracellular domain of the PLA2R1 antigen 
that is recognized by anti-PLA2R1 antibodies [106, 107]. The immunodominant epitope in PLA2R1 has been found in the terminal domain of PLA2R1 in particular in the cystine rich (CysR) region [107]. This region contains a conformational epitope recognized by $90 \%$ of human antiPLA2R1 autoantibodies [106]. The exact mechanism leading to the modification of structure in CysR region of the PLA2R1 antigen remains unknown. However, the structural model gave rise to an interesting pathophysiological molecular mimicry hypothesis linking chronic bacterial infection and pMN [106]. It has been proposed that bacterial infection primes production of autoantibodies able to recognize the dominant epitope in PLA2R1 antigen on podocytes.

Moreover, an additional immune epitope within the PLA2R1 antigen associated with poor outcome of disease has been detected in the C-type lectin domains 1 and 7 (CTLD1 and CTLD7) [108]. The kinetic of immunization starts probably with the production of anti-PLA2R1 antibodies against the epitope in the CysR region, which is the most distal epitope, and is followed by immunization against epitope CTLD1 and finally CTLD7. This epitope spreading of antibody specificity from CysR, CysR + CTLD1 to CysR + CTLD1 + CTLD7 is associated with aging, increases in proteinuria, and poor outcome. On the contrary, in a small retrospective cohort of PLA2R1 related MN patients, the shift over time of anti-PLA2R1 antibody reactivity from a "broad" spectrum against CysR + CTLD1 + CTLD7 epitopes to the single CysR epitope was associated with better clinical outcome [109]. This observation raises the interesting perspective that treatment effects might be evaluated by the evolution of epitope spreading in addition to simple assessment of blood levels of circulating antibodies. However, this strategy has to be validated in prospective trials before implementation in routine clinical practice.

Recently, low levels of regulatory $\mathrm{T}$ cells and increases in circulating plasmablasts as well as in plasma cells have also been suggested as an additional new biomarker of $\mathrm{MN}$ activity and responsiveness to treatment [110-113].

6.4. Anti-Thrombospondin Type 1 Domain Containing 7 Autoantibodies. Recently, it has been reported that 15 out of 154 (10\%) patients with PLA2R1-negative idiopathic MN had detectable anti-THSD7A autoantibodies. As for anti-PLA2R1 antibodies, the circulating serum anti-THSD7A antibodies were mainly IgG4 subtype but a weak presence of other subtypes has also been found [5].

Likewise PLA2R1 related MN, the prevalence of THSD7A related $\mathrm{MN}$ depends on ethnicity, material tested (serum or kidney biopsy), and technique used for the detection of circulating antibodies $[5,7,9,12,17,86,114,115]$. Considering iMN patients, the prevalence of THSD7A related $\mathrm{MN}$ is more frequent (9.1\%) in Japan [7] as compared with European cohort (2\%, 4 out of 198 iMN patients) [115]. Along the same line, among 1276 patients with MN provided from combined cohorts (prospective and retrospective cohort from Hamburg and retrospective cohort from Boston) only 40 patients had detectable antibodies against THSD7A, indicating that THSD7A related $\mathrm{MN}$ is a very rare disease (3.1\%) [17].
The prevalence of anti-THSD7A related MN among patients seronegative for anti-PLA2R1 antibodies appears to be higher in Asian countries (16\% in China [116] and 19\% in Japan [7]) as compared to those reported in the European and Boston cohorts (14\% and 8\%, resp.) [5].

In an intriguing paper from Japan, 8 patients with THSD7A related MN developed a malignancy within 3 months of follow-up. In this study, THSD7A related MN showed female predominance contrasting with the PLA2R1 related $\mathrm{MN}$ which is predominant in men [7].

The THSD7A related MN associated with malignancy has also been recently reported in European and Chinese patients [116, 117]. The possible pathological link between both diseases has been demonstrated by Hoxha et al. in a case of MN in a woman with gallbladder carcinoma [117]. Indeed, the expression of THSD7A antigen by tumor cells in the gallbladder and metastatic cells in lymph nodes suggested the hypothesis that anti-THSD7A antibodies were primed by malignancy secondary leading to the formation of immune complex within the subepithelial deposits in the glomerulus responsible for proteinuria. The chemotherapy resulted in the progressive decrease of circulating anti-THSD7A antibodies and concomitantly a decrease of proteinuria. Recently, highly variable expression of THSD7A with different staining patterns within different malignant cells types has been reported [118].

\section{PLA2R1 and THSD7A Antigens Characteristics and Immunohistochemical Data}

The PLA2R1 and THSD7A antigens are N-glycosylated proteins. PLA2R1 belongs to the superfamily of the lectin of type $\mathrm{C}$ receptors. It stimulates the endocyclic recycling involved in the clearance of soluble phospholipase A2. This enzyme is recognized as a potent inflammatory mediator $[119,120]$. PLA2R1 is involved in cell senescence (apoptotic death) related to mitochondrial superoxide production [121]. In vitro, soluble PLA2R1 receptor induces podocyte apoptosis through ERK1/2 and CPLA2 alpha signaling pathway [122].

The THSD7A is the most extensively characterized member of a family of extracellular matrix glycoproteins involved in the regulation of cellular behavior during tissue genesis and repair [123]. It interacts with glycosaminoglycans, calreticulin, and integrins regulating cellular adhesion in the extracellular environment. THSD7A mediates interaction of low-density lipoprotein receptor during its uptake and clearance at the surface of various cells and also regulates the interaction with fibrinogen during platelet aggregation.

The expression of PLA2R1 and THSD7A antigens is different in terms of localization, pattern, and intensity. In healthy controls the expression of PLA2R1 antigen is confined to the external side of the GBM with typically granular but relatively weak staining, while the THSD7A antigen is expressed within the GBM with a typical linear pattern and more intensely than PLA2R1 [9]. The expression of PLA2R1 is limited to podocytes in humans and not found in rodents [124]. 
The THSD7A antigen-staining pattern is very similar to that of nephrin. Under normal conditions in humans THSD7A expression is limited to the slit diaphragm, the podocyte's soma, endocytic compartment, and foot processes. THSD7A is found in rodents, but not on the GBM or on endothelial cells [125].

New heterologous mouse model of THSD7A related $\mathrm{MN}$ has been reported and confirmed the pathogenicity of THSD7A antibodies in the development of MN lesions [126].

Beck Jr. et al. using immunofluorescence method were first to demonstrate the presence of PLA2R1 antigen within extramembranous glomerular deposits [4]. The circulating anti-PLA2R1 antibodies have been detected by western blot in his work.

Commercial anti-PLA2R1 antibodies are used to identify the PLA2R1 antigen within extramembranous deposits in paraffin sections by an immunohistochemical technique [127]. Interestingly, this technique can be used retrospectively to diagnose PLA2R1 related MN in patients under immunosuppression who no longer have detectable circulating antibodies [47, 127]. This test can also be used to determine whether the PLA2R1 antigen is present in the native kidneys of candidates for renal transplantation, which is important to assess the risk of relapse [128]. Antigen detection within subepithelial deposits is more sensitive (86\%) than detection of corresponding circulating antibodies (76\%) at the time of kidney biopsy [129]. Positive staining for PLA2R1 in glomeruli strongly correlates with the presence of PLA2R1 antibodies in the serum [11].

\section{Anti-PLA2R1 and Anti-THSD7A Antibodies and Primary MN}

8.1. Specificity of Anti-PLA2R1 and Anti-THSD7A Antibodies in Primary MN. The discovery of anti-PLA2R1 and antiTHSD7A antibodies constitutes a major step forward in the management of patients with iMN, as both antibodies are very reliable diagnostic and prognostic markers of disease [10].

Anti-PLA2R1 antibodies are detected in approximately 70 to $80 \%$ of patients with iMN in Europe [130], the USA [4], and Asia [131], with the exception of Japan where the prevalence of these antibodies is lower than in other Asian countries (about $50 \%$ of patients with iMN) $[8,132]$.

The detection of anti-PLA2R1 antibodies in patients with nephrotic syndrome can be considered as a biomarker for the diagnosis of primary $\mathrm{MN}$ according to a recent meta-analysis (all study sensitivity 78\% (95\% CI: 66\% to 87\%) and specificity 99\% (95\% CI: $96 \%$ to $100 \%)$ ) [6]. This important evidence can modify the indication for kidney biopsy in specific situations such as patients with a single kidney or those with increased risk of bleeding [14].

In addition to their significant sensitivity, the specificity of anti-PLA2R1 antibodies for $\mathrm{MN}$ has been proposed close to $100 \%$ as they have not been detected in healthy subjects and in non-MN glomerular diseases [4]. However, even if their prevalence is much lower, the presence of anti-PLA2R1 antibodies in secondary MN (active sarcoidosis, lupus nephritis, and $\mathrm{HBV}$ infection) has been reported [32, 33, 38-52]. However, the occurrence of anti-PLA2R1 autoantibodies in membranous lupus nephritis is very rare and they have never been observed in non-MN lupus nephritis. Recently, circulating anti-PLA2R1 antibodies have been reported in patients that had IgA nephropathy [133]. In retrospective study of 26 patients with biopsy-proven glomerular lesions that occurred in patient with sarcoidosis, $\mathrm{MN}$ preceded or occurred concomitantly with active sarcoidosis but was not reported in inactive sarcoidosis [46]. The expression of PLA2R1 antigen has been found in glomerular deposits in MN patients with sarcoidosis [45, 49]. Moreover, the PLA2R1 antigen within the glomerular deposits in $7.7 \%$ to $64 \%$ [61, 134] of a Chinese series of secondary MN related to $\mathrm{HBV}$ infection and was associated with presence of circulating antiPLA2R1 antibodies [134].

Recent reports demonstrated that anti-THSD7A and antiPLA2R1 MN are not mutually exclusive [9] as it has been previously proposed [5]. Described by Larsen et al. for the first time, double PLA2R1 and THSD7A MN is a very rare condition $(0.7 \%$ of incident $\mathrm{MN})[9,116]$.

8.2. Anti-PLA2R1 and Anti-THSD7A Antibodies, Biomarkers of Disease Activity, Clinical Outcome, and Treatment Efficacy. Circulating anti-PLA2R1 antibodies reflect immunological activity of disease $[4,135]$ and have been shown to disappear before clinical remission of nephrotic syndrome [4] and to reappear in the circulation before clinical relapse [23].

Serum anti-PLA2R1 antibodies levels correlate with the degree of proteinuria and the reduction or resolution of antiPLA2R1 antibodies levels has been shown to precede by at least 9 months of partial or complete remission proteinuria [135]. These data were confirmed by larger cohorts, which also found a correlation between anti-PLA2R1 antibodies levels and both the remission rate and the time to double serum creatinine $[13,15]$.

As compared to patients with non-PLA2R1 related $\mathrm{MN}$, those with PLA2R1 related $\mathrm{MN}$ responded slower to immunosuppressive therapy $[135,136]$. Moreover, patients presenting high titer of autoantibodies have more severe disease and a longer time to disease remission [137]. These observations illustrate the important role of anti-PLA2R1 antibodies levels as a prognostic marker of long-term clinical outcome of MN.

Similar data demonstrating that the disappearance of anti-THSD7A antibodies precedes remission of proteinuria and that an increase in the titer anti-THSD7A predicts the reappearance of proteinuria in a patient with a relapse has been reported in Chinese cohort [116].

8.3. Anti-PLA2R1 and Anti-THSD7A Antibodies, Biomarkers for Relapse of MN following Kidney Transplantation. To date, positive expression of PLA2R1 antigen has only been detected exceptionally in "de novo" cases as compared with recurrent $\mathrm{MN}$ in kidney transplant patients (8\% versus $83 \%)[138,139]$. Nearly $50 \%$ of cases of recurrent $\mathrm{MN}$ on the kidney graft are associated with the presence of anti-PLA2R1 antibodies $[128,140,141]$. The detection or persistence of anti-PLA2R1 in kidney transplant patients has been associated with an 
increased risk of loss of graft function [12, 95, 139]. Recently, the recurrence of THSD7 A related $\mathrm{MN}$ has been also reported and raises the issue of monitoring anti-THSD7A antibodies after renal transplantation [86]. However, it is actually unknown if relapses are more frequent in patients with related anti-THSD7A. Despite a broad consensus that assessment of anti-PLA2R1 in iMN and anti-THSD7A antibodies in antiPLA2R1 serum negative iMN patients is indicated there is still no consensus on the frequency of assays during follow-up.

\section{Anti-PLA2R1 and Anti-THSD7A Antibodies: Serological Detection Methods}

Techniques allowing for the detection and measurement of anti-PLA2R1 antibodies have developed very rapidly. Initial Western blots using protein extracts of human kidneys [4] or extracts of cells transfected with recombinant human cDNA for PLA2R1 have been replaced with a commercial bioassay using a recombinant cell-based indirect immunofluorescence assay (RC-IFA) technique [13] on slides containing "biochips" coated with human embryonic kidney cells (HEK 293) transfected with recombinant human cDNA PLA2R1 or THSD7A and nontransfected human embryonic kidney cells (HEK 293) (Figures 3(a) and 3(b)) [17]. The patient serum samples are incubated with increasing dilutions and the results are expressed as titers, similar to antinuclear antibodies. Comparison of the RC-IFA and western blots in a series of 42 cases revealed a very good qualitative correlation between the two tests $(100 \%)$, with some quantitative discrepancies. Recently an immunoenzymatic method (ELISA) developed and is more quantitative than RC-IFA [13]. The addressable laser bead immunoassay (ALBIA) technique has been adapted for the detection of PLA2R1 antibodies and, in addition to its good diagnostic performance, ALBIA offers the benefit of multiplex analysis of other nephrotoxic antibodies and/or other immunological markers (Figure 3) [96]. A recent metaanalysis provided very high specificity and sensitivity of antiPLA2R1 antibodies for the detection of $\mathrm{pMN}$ (99\% and 78\%, resp.) [6].

\section{Other Potentially Pathogenic Antibodies}

Antibodies against cytoplasmic alpha-enolase have long been known to be present in the serum of patients with primary and secondary MN (nearly 70\%) [142] but never within the subepithelial deposits [143]. Antibodies against other cytoplasmic podocyte proteins, including aldose reductase and manganese superoxide dismutase 2, have also been detected in the serum and tissue eluates of microdissected glomeruli in biopsy samples from patients with MN [144]. These cytoplasmic antigens are not accessible to circulating antibodies under normal conditions. However, in the event of oxidative stress, they can migrate to the cell membrane and serve as targets for circulating antibodies. The activation of complement leading to the formation of MAC is a cause of oxidative stress in podocytes [71]. The initial lesions induced by the immune complexes involving PLA2R1 may induce oxidative stress responsible for this novel membrane expression of proteins, which are physiologically cytoplasmic, leading to the formation of new autoantibodies [10]. Their pathogenic role in the initiation and/or maintenance of the disease is still hypothetical.

\section{Treatment of MN: Current Controversies}

MN presents in a large spectrum of disease severity and has the potential for spontaneous remission without therapy and its progression is difficult to predict, which explains the therapeutic uncertainties that still persist today [145-147]. In less than $30 \%$ of the cases, the disease progresses slowly towards severe renal insufficiency despite optimal supportive care combined with classical immunosuppressive therapy $[19,22$, 30, 147]. According to the KDIGO guidelines, the predictive factors for poor prognosis of $\mathrm{MN}$ are a decrease in glomerular filtration rate at the time of diagnosis, persistent nephrotic proteinuria at 6 months of optimal nephroprotection, male sex, age over 50 years, uncontrolled arterial hypertension, and the presence of interstitial fibrosis and tubular atrophy seen on a renal biopsy $[148,149]$.

11.1. Optimal Nephroprotection. Patients with nonnephrotic proteinuria $(<3.0 \mathrm{~g} /$ day) without other symptoms such as increase in serum creatinine level or uncontrolled arterial hypertension are in general managed by optimal supportive care alone [147]. Optimal supportive care of MN consists of a standard renoprotective treatment (drugs that inhibit the renin-angiotensin-aldosterone system, control of arterial hypertension, dyslipidaemia, excess weight, and other cardiovascular risk factors) [148]. In a recent retrospective cohort study of patients with iMN in the absence of anti-PLA2R1 or anti-THSD7A antibodies and receiving optimal supportive care the 24-month outcomes were similar irrespective of the administration of immunosuppressive therapy [115], confirming previous reports of favorable evolution of some patients in the absence of immunosuppressive therapy [150].

11.2. Immunosuppressive Therapy. In 2017, the best therapeutic approach of MN still remains debated and varies between countries [151, 152]. Immunosuppressant therapies are in general administered to patients at risk of progression to the end-stage kidney disease (ESKD) based on persistent proteinuria for more than 6 months and/or impaired kidney function at the time of diagnosis of the disease [151-153]. Indeed, $86 \%$ renal survival at 10 years has been reported in a cohort of patients in whom immunosuppressive therapy based predominantly on cyclophosphamide and steroids was selectively administered because of bad renal prognostic [154]. Absence of remission or relapse following partial remission has been reported to be significantly associated with progression to stage 5 of chronic kidney disease [155]. Cytotoxic immunosuppressive treatment should be reserved for those forms with refractory nephrotic syndrome or renal insufficiency [154] as it is associated with a high rate of complications over the short term [neutropenia, anaemia, and thrombocytopenia] as well as the long term (neoplasms). Cyclophosphamide is preferred because of being less toxic than chlorambucil [156]. 
Recombinant cell-based indirect immunofluorescence assay (RC-IFA)

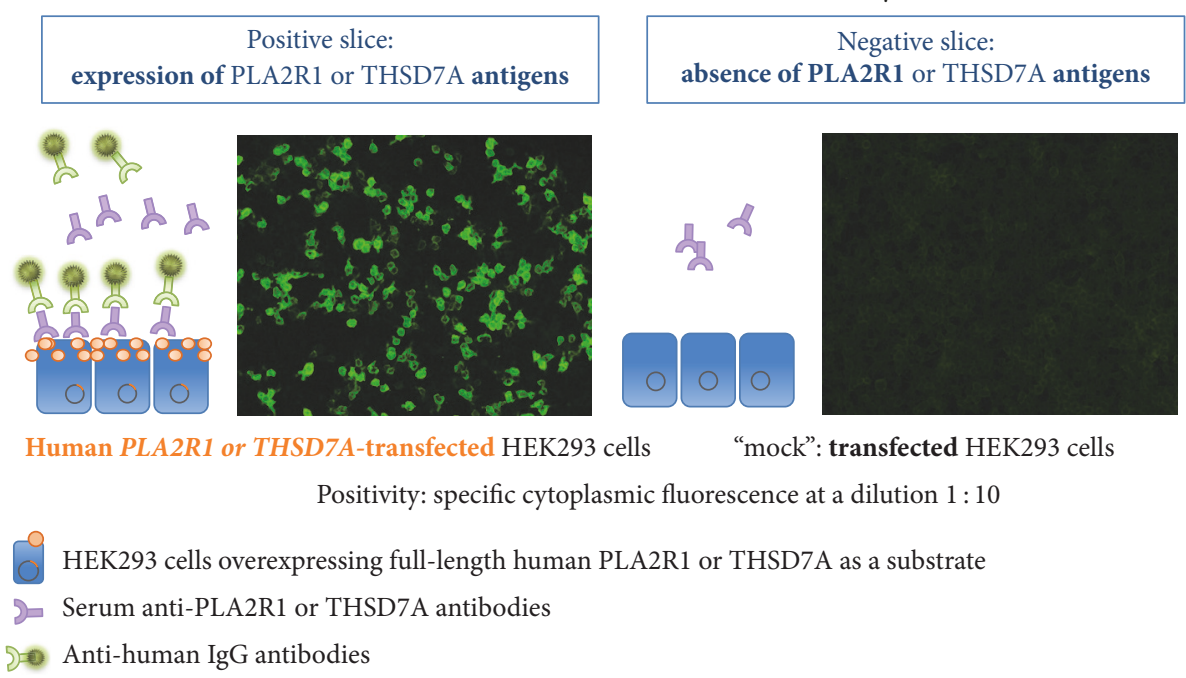

(a) Anti-PLA2R1 and anti-THSD7A antibodies immunoassay for detection and measurement

Enzyme-linked immunosorbent assay (ELISA)

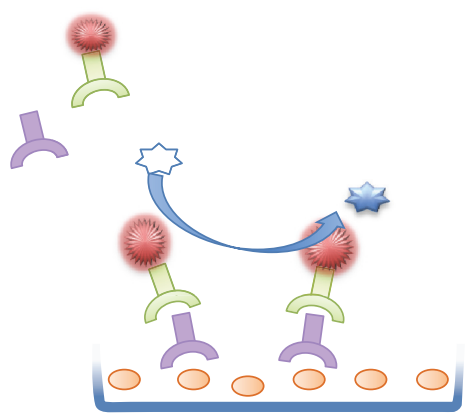

○ PLA2R1

? $=$ Anti-PLA2R1 antibodies

$D=0$ Enzyme labelled anti-IgG4 antibodies
Addressable laser bead immunoassay

(ALBIA)

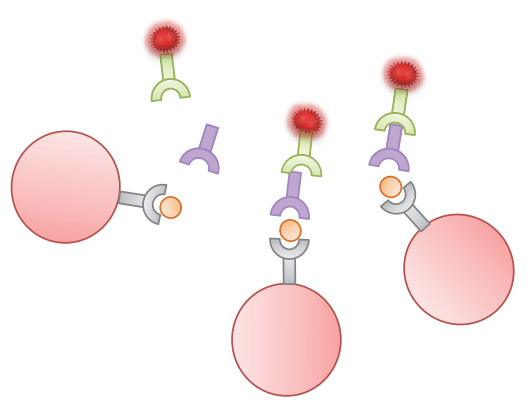

\{ 3 Colorant substrate

Bead labelled by PLA2R1 antigen

(b) Anti-PLA2R1 Ab immunoassay for detection and measurement

FIGURE 3: Schematic presentations of available immunoassays detecting circulating anti-phospholipase 2 receptor 1 (PLA2R1) and antithrombospondin type 1 domain containing 7A (THSD7A) autoantibodies for diagnostic of primary membranous nephropathy (pMN). (a-b) Three standardized assays are currently available for diagnostic purposes of primary MN. The assessment of PLA2R1 could be performed using recombinant cell-based indirect immunofluorescence assay (RC-IFA), enzyme-linked immunosorbent assay (ELISA), or addressable laser bead immunoassay (ALBIA). The RC-IFA and ELISA are highly suitable for routine evaluation of pMN patients and are commercialized worldwide (Euroimmun AG, Luebeck, Germany). The ALBA developed by Mitogen Advanced Diagnostic Laboratory, Calgary, Canada, is a promising technique as it offers the possibility of analysing several antibodies in the same samples by one test. The RC-IFA uses the HEK293 human cell line overexpressing full-length human PLA2R1 protein. RC-IFA is a biochip format containing in one incubation field cells that express PLA2R1 antigen and control-transfected cells incapable of expressing PLA2R1. Using this test, anti-PLA2R1 antibodies are detected with very high specificity (nearly 100\%) and high sensitivity (77\%). Titers of anti-PLA2R1 antibodies decline during successful immunosuppressive therapy as well as during the spontaneous remission.

The efficacy of calcineurin inhibitors (cyclosporine and tacrolimus) used alone has been demonstrated by a large number of studies and confirmed by recent meta-analysis (2018 patients from 35 randomized controlled trials) [148, 156-160]. The high rate of remission of proteinuria contrasts the significant relapse rate after cessation of therapy $(91 \%$ and $42 \%$, resp.) [161]. In this study, $40 \%$ of patients with the highest proteinuria at 3-month follow-up and the highest time-averaged proteinuria were more likely to develop cyclosporine-induced acute kidney injury and to reach an 
adverse renal endpoint. In these patients switch to a regimen without calcineurin inhibitors has been proposed as a reasonable alternative.

Mycophenolate mofetil may be beneficial when associated with steroids [147]. MMF significantly reduced proteinuria but had no significant effect on the induction of complete remission and was also associated with an increased risk of relapse $[162,163]$.

Anti-CD20 antibodies [164] and adrenocorticotropic hormone (corticotropin) [165] offer new therapeutic opportunities that appear to be promising. A novel therapy based on proteasome inhibitor has been used with benefit in rituximab-resistant or partially responsive recurrent posttransplant membranous nephropathy [113].

The addition of the anti-CD20 antibody to the classical immunosuppressant treatment has been proposed to block the proliferation of $\mathrm{B}$ cells and the production of pathogenic antibodies in patients with recurrent $\mathrm{MN}[140,166,167]$.

Anti-PLA2R1 antibodies are valuable tools for the evaluation of treatment efficacy. Remission of nephrotic syndrome induced by RTX has been significantly associated with decrease in levels of circulating anti-PLA2R1 antibodies at 6 and 12 months [94] and after 24 months [135]. In the prospective randomized GEMRITUX trial, higher remission rate of proteinuria has been associated with decrease in circulating anti-PLA2R1 antibodies levels at 6 months after RTX [168]. Low anti-PLA2R1 antibodies level before treatment initiation or their absence and high albuminemia at 3 months were also significantly associated with remission in RTX treated patients. Adjunction of RTX to antiproteinuric standard therapy induced a higher remission rate of proteinuria after 6 months of randomization (35.1\% versus 21\%). The disappearance of circulating anti-PLA2R1 antibodies in $82 \%$ of patients preceded the increase of serum albumin level and the reduction in proteinuria [129] demonstrating the value of anti-PLA2R1 as a new helpful immunological biomarker for assessment of disease activity and treatment efficacy. Indeed, B cell-depleting strategies promote proteinuria reduction and clearance of serum anti-PLA2R1 autoantibodies [169172]. Anti-CD20 monoclonal antibodies have been suggested as a first line of therapy for patients with iMN at risk of progression [112]. Indeed RTX is safe and noninferior to cyclosporine as long-term proteinuria remission has been reported in patients with iMN [173].

Retrospective analysis and prospective studies as well as randomized controlled trial have reported significant remission rate of nephrotic syndrome (65\%) in patients with MN after RTX [129]. There is no consensus on the optimal regimen of RTX to treat $\mathrm{MN}$ and a variety of doses and administration frequencies have been used in different studies [174]. The safety profile has been confirmed in several studies; however the physician must be aware of rare but possible risk of acute nonischemic cardiomyopathy [175] and progressive urticarial dermatitis [176] related to the RTX administration.

Some authors suggest that depletion of peripheral B cells could be insufficient to ensure sustained remission; therefore higher doses and longer treatment duration could be considered when RTX is used to prevent the relapses $[177,178]$.

However, if more intensive treatment regimens have the potential to improve efficacy of RTX this has to be balanced against a higher risk of adverse effects and increased costs and should be evaluated by prospective trials. Waiting for the results from such clinical trials the $\mathrm{B}$ cell-driven protocol is proposed as the preferential regimen for RTX administration [174].

A new protocol based on the association of RTX and cyclosporine for 6 months, followed by a second cycle of RTX and tapering of cyclosporine during 18 months as a maintenance phase, demonstrated that proteinuria decreased by $80 \%$ at 6 months. Moreover complete remission has been reported in 54\% at 12 months [179].

A novel therapy based on proteasome inhibitor (bortezomib, 4 doses of $1.3 \mathrm{mg} / \mathrm{m}^{2}$ over 2 weeks) efficiently reduced proteinuria in a case of recurrent $\mathrm{MN}$ after renal transplantation that had been only partially responsive to RTX [180].

In addition to rituximab, the development of new monoclonal antibodies targeting B cells such as ofatumumab and belimumab as well as new treatments such as bortezomib and eculizumab provides a variety of potential therapies with the potential to ultimately replace the nonspecific and toxic immunosuppressants that are the current standard of care of pMN [181]. In the near future treatment protocols for $\mathrm{MN}$ patients will probably be individualized based on the level of anti-PLA2R1 antibodies and proteinuria [182].

\section{Actualized Workup of MN}

A growing number of studies have generated strong evidence which calls for the adaptation of management of $\mathrm{MN}$ in the light of the present state of knowledge. The discoveries of anti-PLA2R1 anti-THSD7A antibodies literally shifted the paradigms in clinical workup of patients with $\mathrm{MN}$, leading to the introduction of new terminology which is PLA2R1 or THSD7A related MN (Figure 4) [183]. High antiPLA2R1 antibody levels have recently been reported as a new promising prognostic factor [13-16] and will probably modify the indications of treatment and how the disease is managed to improve the health and long-term outcomes of patients with MN. The present state of knowledge on antipodocyte antibodies in the pathogenesis of $\mathrm{MN}$ and their role in monitoring disease activity and response to treatment is not reflected in international guidelines.

Awaiting the recommendations form revised KIDGO guidelines we propose a revised clinical workup for patient with MN. The previously proposed specificity of IgG4 expression within the deposits for $\mathrm{pMN}$ remains matter of debate. In contrast, the screening for the presence of circulating antibodies against PLA2R1 [4] and THSD7A [5], useful new tools reflecting autoimmune activity, is crucial to differentiating the "pure pMN" case from secondary MN. It is only necessary to test for anti-THSD7A in those patients who have apparently primary $\mathrm{MN}$ and who are negative for anti-PLA2R antibodies. Moreover, the routine investigation of kidney tissue biopsy needs to include the histological evaluation of glomerular immune deposits for PLA2R1 [53, 184], cationic 


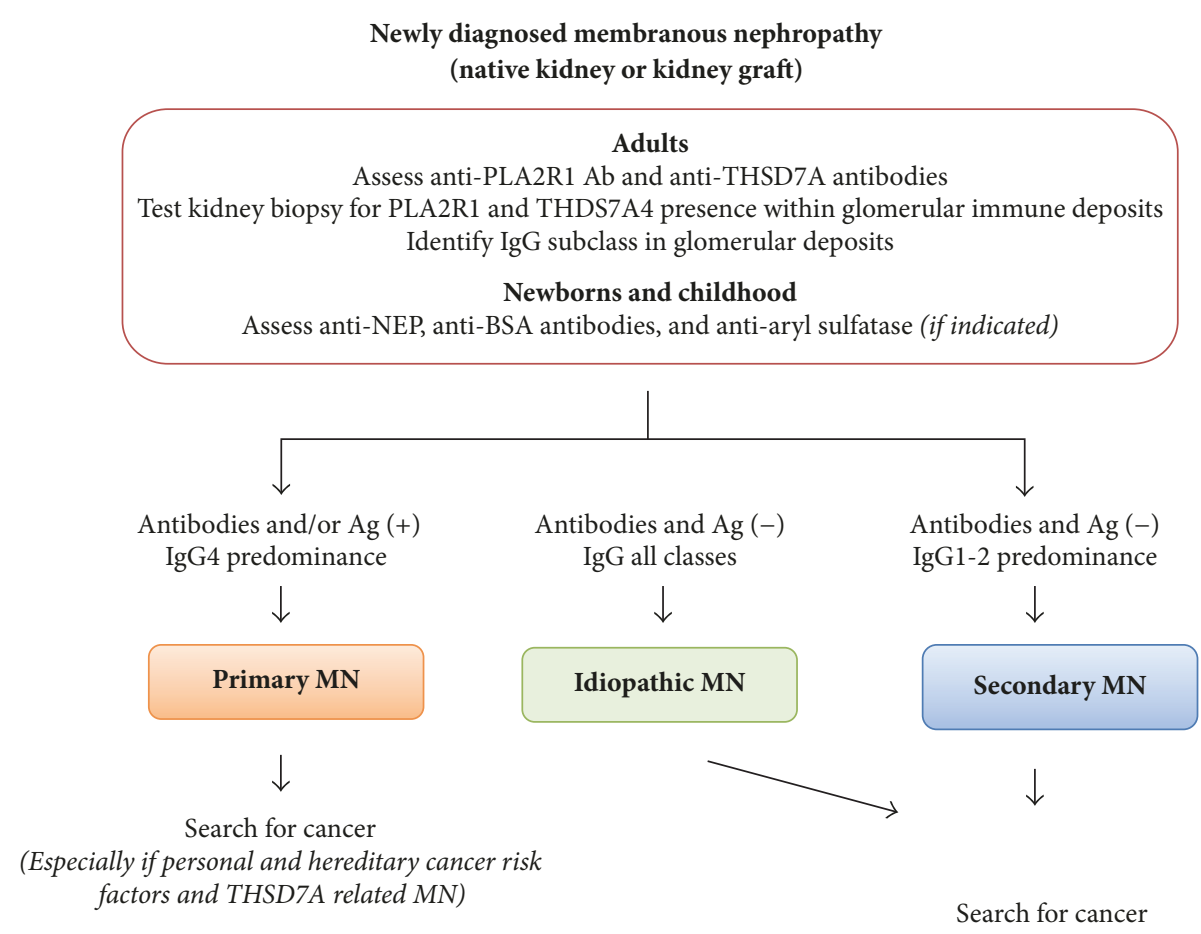

Optimal nephroprotection during 6 months

Secondary diseases therapy if secondary causes associated

High levels of antibodies at 6 to 9 months consider immunosuppression

Optimal nephroprotection Secondary diseases therapy

FIGURE 4: Proposed summarized workup for diagnostic of membranous nephropathy in native kidney and kidney graft. We propose a revised clinical workup for patients with MN. The screening for the presence of circulating antibodies against PLA2R1 and THSD7A, useful new tools reflecting that autoimmune activity is essential for differentiating "pure pMN" from secondary MN. It is only necessary to test for anti-THSD7A in those patients who have apparently primary MN and who are negative for anti-PLA2R antibodies. Moreover, the routine investigation of kidney tissue biopsy needs to include the histological evaluation of glomerular immune deposits for PLA2R1, cationic BSA in childhood, and aryl sulfatase in patients under enzymes replacement therapy. Testing for THSD7A antigen is only required in cases with suspected primary MN that is negative for PLA2R1 antigen or who are suspected of having cancer-related MN. Those histological biomarkers are far more specific for pMN than the presence of less than 8 inflammatory cells per glomerulus. Under these circumstances further etiological investigations could by stopped except in patients with important personal and hereditary risk factors for cancer and management focused on optimal nephroprotective and immunosuppressive therapy. Intensive screening for secondary causes especially for the presence of malignancies is still advised in patients with THSD7A related MN. On the contrary, the absence of circulating antiPLA2R1 antibodies and/or PLA2R1 antigen in association with IgG1 or IgG2 subclasses within extramembranous deposits is associated with an increased likelihood of secondary MN related to cancer, which should be thoroughly excluded by extensive investigation. In this setting, the treatment of secondary causes and optimal nephroprotection without immunosuppressive therapy has been advised. The overlap of pMN and secondary $\mathrm{MN}$, especially in sarcoidosis or HBV infection, is possible. It could suggest postponing immunosuppression in patients with HBV infection and focusing on antiviral therapy. In this situation, monitoring of anti-PLA2R1 antibodies could be helpful to assess renal response. The levels of circulating antibodies need to be regularly monitored during the follow-up to evaluate the long-term outcome of disease, efficacy of therapeutic interventions, and the risk of disease relapse or of recurrence in recipients of kidney transplants. Adjustments of interventions in function of the evolution of antibody levels are likely to have a central role in individualizing care of patients with pMN in the future. Persistence over 6 to 9 months or increase in antibodies level associated with nephrotic proteinuria is a strong argument in favor of adjunction of immunosuppression to optimal supportive care. It would be logical to start when antibody levels are high to prevent worsening proteinuria and, on the contrary, to stop or at least taper the immunosuppressive treatment once the anti-PLA2R1 antibody levels are no longer detectable, which occurs in general months before urine protein levels decrease.

BSA [2] in childhood, and aryl sulfatase in patients under enzymes replacement therapy [185]. Testing for THSD7A antigen is only needed in cases of suspected primary $\mathrm{MN}$ that is negative for PLA2R1 antigen or cases suspected of having cancer-related MN. Those histological biomarkers are far more specific for pMN than the presence of less than 8 inflammatory cells per glomerulus [65] (Figure 4) [3].

Under these circumstances further etiological investigations could be stopped except in patients with important personal and hereditary risk factors for cancer and management 
focused on optimal nephroprotective and immunosuppressive therapy [184]. Intensive screening for secondary causes especially for the presence of malignancies is still actually advised in patients with THSD7A related MN [17].

On the contrary, the absence of circulating anti-PLA2R1 antibodies and/or PLA2R1 antigen associated with predominantly IgG1 or IgG2 subclasses within extramembranous deposits is associated with an increased likelihood of secondary MN related to cancer, which should be thoroughly excluded by extensive investigation. In this setting, the treatment of secondary causes and optimal nephroprotection without immunosuppressive therapy has been advised [10, $148,184]$.

The overlap of pMN and secondary MN, especially in sarcoidosis or HBV infection, is possible. Those observations could be simply interpreted as an unrelated coincidence of two diseases by some authors. An alternative hypothesis is that active sarcoidosis or chronic HBV infection prompts an immune response allowing the development of antiPLA2R1 antibodies. This scenario has potential practical implications for the treatment strategy. It could suggest postponing immunosuppression in patients with $\mathrm{HBV}$ infection and focusing on antiviral therapy [35]. In this situation, monitoring of anti-PLA2R1 antibodies could be helpful to assess renal response.

The levels of circulating antibodies need to be regularly monitored during the follow-up to evaluate the long-term outcome of disease, efficacy of therapeutic interventions, and the risk of disease relapse or of recurrence in recipient of kidney transplants. Adjustments of interventions in function of the evolution of antibody levels is likely to have a central role in individualizing care of patients with $\mathrm{pMN}$ in the future.

Persistence over 6 to 9 months or increase in antibodies level associated with nephrotic proteinuria is strong argument in favor of adjunction of immunosuppression to optimal supportive care. Indeed, higher levels of circulating antiPLA2R1 antibodies strongly correlate with the importance of proteinuria, predict a higher risk of recurrence of nephrotic syndrome and decline of renal function, and are associated with a lower rate and a longer time to obtain remission. These observations raise the question as to the best time to start and to discontinue immunosuppressive treatment. It would be logical to start when antibody levels are high to prevent worsening proteinuria and, on the contrary, to stop or at least taper the immunosuppressive treatment once the anti-PLA2R1 antibody levels are no longer detectable, which occurs in general months before urine protein levels decrease. Timely reduction of the intensity of immunosuppression should reduce the risk of overimmunosuppression and treatmentrelated side effects [168]. However, such a strategy of tailored immunosuppression remains to be validated in prospective studies.

In the near future nonspecific and toxic immunosuppressive treatments will undoubtedly be increasingly replaced by more specific and less toxic interventions directly targeting the key autoimmune disease mechanisms by targeting B cell proliferation and antibody production [182].

\section{Conclusions}

The recent discovery of an autoimmune mechanism related to anti-PLA2R1 and anti-THSD7A antibodies in adults and a fetomaternal alloimmunization against NEP in neonates has considerably improved our understanding of the pathogenesis of MN. Detection of anti-PLA2R1 and anti-THSD7A antibodies in serum and of the PLA2R1 and THSD7A antigens in subepithelial immune deposits constitutes a major breakthrough in the management of patients with MN.

Current research focuses on the pathophysiological mechanisms responsible for the production of these antibodies and their role in the process leading to podocyte lesions and interstitial renal fibrosis. Independently of the proteinuria, the anti-PLA2R1 and anti-THSD7A antibodies levels represent useful biomarkers for the diagnosis of $\mathrm{MN}$ and for monitoring the efficacy of therapeutic interventions. Approximately $50 \%$ of the cases of recurrent $\mathrm{MN}$ following kidney transplantation are associated with the presence of anti-PLA2R1 antibodies. However, the presence of these antibodies at the time of the transplantation is not constantly associated with a relapse. Anti-PLA2R1 epitope spreading emerged as new promising prognostic biomarkers for clinical outcomes. The systematic testing for a secondary cause including malignancy still needs to be performed, in particular in patients with THSD7A related $\mathrm{MN}$.

We suggest that, after systematic screening for classical secondary cause of MN, the analysis of PLA2R1 and THDS7A (especially in MN associated with cancer) is indicated. Routine investigation should include screening for antibodies against PLA2R1 and THSD7A in anti-PLA2R1 seronegative $\mathrm{MN}$ and the corresponding target antigens in kidney tissue in adults, whereas deposits of NEP should be excluded in newborns as well as cationic BSA and aryl sulfatase during childhood. Despite the demonstration of efficacy and safety of new specific therapies targeting B cells (anti-CD20 antibodies, inhibitors of proteasome) in pMN, their place as first-line immunosuppression remains to be defined in treatment guidelines for $\mathrm{MN}$ that need to be updated in the light of the important evidence generated during the last few years. In the near future nonspecific and toxic immunosuppressive treatment will undoubtedly be progressively replaced by the immunosuppressive regimens incorporating selective inhibitors of $\mathrm{B}$ cell proliferation and antibody production.

\section{Additional Points}

Clinical Summary. (i) The prevalence of anti-PLA2R1 and anti-THSD7A antibodies is approximately $70 \%$ and $2 \%$ in adults with idiopathic membranous nephropathy. (ii) Assessment of glomeral expression of PLA2R1 and of THSD7A antigen in case of PLA2R1 seronegative $\mathrm{MN}$ deposits is at present mandatory in idiopathic membranous nephropathy. (iii) The presence of anti-PLA2R1 antibodies has been reported in membranous nephropathy associated with secondary conditions (hepatitis B virus, membranous form of lupus nephritis, sarcoidosis, or malignancy); however their significance remains a matter of debate. (iv) The absence of anti-PLA2R1 
in patients without immunosuppressive treatment and/or the absence of PLA2R1 expression within immune deposits should prompt systematic testing for a secondary cause of $\mathrm{MN}$, including neoplasms, in particular in patients over the age of 60. Such a screening is also indicated in patients with anti-THSD7A related MN. (v) Circulating anti-PLA2R1 antibodies are considered valid biomarkers of immunological activity that correlate with markers of disease activity (degree of proteinuria) and are useful in predicting clinical outcomes such as response to treatment, long-term kidney function, and disease recurrence. (vi) Approximately 50\% of cases of recurrent membranous nephropathy after kidney transplantation are associated with anti-PLA2R1 antibodies. (vii) Recently, epitope spreading within anti-PLA2R1 antibodies, levels of regulatory $\mathrm{T}$ cells, circulating plasmablasts, and plasma cells emerged as interesting additional tools for monitoring of immunological activity in primary membranous nephropathy. (viii) New heterologous mouse models of antiTHSD7A4 related $\mathrm{MN}$ are available at present. (ix) The monitoring of circulating anti-PLA2R1 and anti-THSD7A antibodies levels is indicated in patients with PLA2R1 or THSD7A-related $\mathrm{MN}$, respectively. However, there is at present no consensus on the optimal monitoring schedule. (x) New specific therapies targeting B cells (anti-CD20 antibodies, inhibitors of proteasome) have demonstrated their efficacy and safety in primary membranous nephropathy, but their use as a first-line immunosuppression is still not recommended according to kidney disease improvement global outcome (KDIGO) 2012 guidelines that need to be revised.

\section{Conflicts of Interest}

The authors declare no conflicts of interest.

\section{Acknowledgments}

The authors express their gratitude to M-C Endlé (Biognost, Heule, Belgium) for providing them with the picture of anti-PLA2R1 antibodies detection by recombinant cell-based indirect immunofluorescence assay (RC-IFA).

\section{References}

[1] H. Debiec, V. Guigonis, B. Mougenot et al., "Brief report: Antenatal membranous glomerulonephritis due to anti-neutral endopeptidase antibodies," The New England Journal of Medicine, vol. 346, no. 26, pp. 2053-2060, 2002.

[2] H. Debiec, F. Lefeu, M. J. Kemper et al., "Early-childhood membranous nephropathy due to cationic bovine serum albumin," The New England Journal of Medicine, vol. 364, no. 22, pp. 21012110, 2011.

[3] H. Debiec, V. Valayannopoulos, O. Boyer et al., "Allo-immune membranous nephropathy and recombinant aryl sulfatase replacement therapy: A need for tolerance induction therapy," Journal of the American Society of Nephrology, vol. 25, no. 4, pp. 675-680, 2014.

[4] L. H. Beck Jr., R. G. B. Bonegio, G. Lambeau et al., "Mtype phospholipase A2 receptor as target antigen in idiopathic membranous nephropathy," The New England Journal of Medicine, vol. 361, no. 1, pp. 11-21, 2009.

[5] N. M. Tomas, L. H. Beck Jr., C. Meyer-Schwesinger et al., "Thrombospondin type-1 domain-containing 7A in idiopathic membranous nephropathy," The New England Journal of Medicine, vol. 371, no. 24, pp. 2277-2287, 2014.

[6] Y. Du, J. Li, F. He et al., "The diagnosis accuracy of PLA2R-AB in the diagnosis of idiopathic membranous nephropathy: A metaanalysis," PLoS ONE, vol. 9, no. 8, Article ID e104936, 2014.

[7] T. Iwakura, N. Ohashi, A. Kato, S. Baba, and H. Yasuda, "Prevalence of enhanced granular expression of thrombospondin type-1 domain-containing 7A in the Glomeruli of Japanese patients with idiopathic membranous nephropathy," PLoS ONE, vol. 10, no. 9, Article ID e0138841, 2015.

[8] S. Akiyama, M. Akiyama, E. Imai, T. Ozaki, S. Matsuo, and S. Maruyama, "Prevalence of anti-phospholipase A2 receptor antibodies in Japanese patients with membranous nephropathy," Clinical and Experimental Nephrology, vol. 19, no. 4, pp. 653660, 2014.

[9] C. P. Larsen, L. N. Cossey, and L. H. Beck, “THSD7A staining of membranous glomerulopathy in clinical practice reveals cases with dual autoantibody positivity," Modern Pathology, vol. 29, no. 4, pp. 421-426, 2016.

[10] D. C. Cattran and P. E. Brenchley, "Membranous nephropathy: integrating basic science into improved clinical management," Kidney International, vol. 91, no. 3, pp. 566-574, 2017.

[11] P. Ronco and H. Debiec, "Pathophysiological advances in membranous nephropathy: Time for a shift in patient's care," The Lancet, vol. 385, no. 9981, pp. 1983-1992, 2015.

[12] J. M. Francis, L. H. Beck, and D. J. Salant, "Membranous Nephropathy: A Journey from Bench to Bedside," American Journal of Kidney Diseases, vol. 68, no. 1, pp. 138-147, 2016.

[13] J. M. Hofstra, H. Debiec, C. D. Short et al., "Antiphospholipase $A_{2}$ receptor antibody titer and subclass in idiopathic membranous nephropathy," Journal of the American Society of Nephrology, vol. 23, no. 10, pp. 1735-1743, 2012.

[14] J. M. Hofstra and J. F. M. Wetzels, "Phospholipase A2 receptor antibodies in membranous nephropathy: unresolved issues," Journal of the American Society of Nephrology, vol. 25, no. 6, pp. 1137-1139, 2014.

[15] D. Kanigicherla, J. Gummadova, E. A. McKenzie et al., "AntiPLA2R antibodies measured by ELISA predict long-term outcome in a prevalent population of patients with idiopathic membranous nephropathy," Kidney International, vol. 83, no. 5, pp. 940-948, 2013.

[16] L. Beck, A. S. Bomback, M. J. Choi et al., "KDOQI US commentary on the 2012 KDIGO clinical practice guideline for glomerulonephritis," American Journal of Kidney Diseases, vol. 62, no. 3, pp. 403-441, 2013.

[17] E. Hoxha, L. H. Beck, T. Wiech et al., "An indirect immunofluorescence method facilitates detection of thrombospondin type 1 domain-containing 7A-specific antibodies in membranous nephropathy," Journal of the American Society of Nephrology, vol. 28, no. 2, pp. 520-531, 2017.

[18] D. T. Erwin, J. V. Donadio Jr., and K. E. Holley, “The clinical course of idiopathic membranous nephropathy," Mayo Clinic Proceedings, vol. 48, no. 10, pp. 697-712, 1973.

[19] R. J. Glassock, "Pathogenesis of membranous nephropathy: a new paradigm in evolution," Contributions to Nephrology, vol. 181, pp. 131-142, 2013. 
[20] W. C. Chan and Y. C. Tsao, "Diffuse membranous glomerulonephritis in children," Journal of Clinical Pathology, vol. 19, no. 5, pp. 464-469, 1966.

[21] S. Menon and R. P. Valentini, "Membranous nephropathy in children: clinical presentation and therapeutic approach," Pediatric Nephrology, vol. 25, no. 8, pp. 1419-1428, 2010.

[22] P. Ronco and H. Debiec, "Advances in membranous nephropathy: Success stories of a long journey," Clinical and Experimental Pharmacology and Physiology, vol. 38, no. 7, pp. 410-416, 2011.

[23] J. M. Hofstra, L. H. Beck Jr., D. M. Beck, J. F. Wetzels, and D. J. Salant, "Anti-phospholipase $A_{2}$ receptor antibodies correlate with clinical status in idiopathic membranous nephropathy," Clinical Journal of the American Society of Nephrology, vol. 6, no. 6, pp. 1286-1291, 2011.

[24] P. Ronco and H. Debiec, "Pathogenesis of membranous nephropathy: Recent advances and future challenges," Nature Reviews Nephrology, vol. 8, no. 4, pp. 203-213, 2012.

[25] P. Ruggenenti and G. Remuzzi, "Idiopathic membranous nephropathy: back to the future?” The Lancet, vol. 381, no. 9868, pp. 706-708, 2013.

[26] R. Mactier, B. Jones, C. D. Payton, and A. McLay, "The natural history of membranous nephropathy in the west of Scotland," QJM: An International Journal of Medicine, vol. 60, no. 2, pp. 793-802, 1986.

[27] T. Toth and S. Takebayashi, "Idiopathic membranous glomerulonephritis: a clinicopathologic and quantitative morphometric study," Clinical Nephrology, vol. 38, no. 1, pp. 14-19, 1992.

[28] G. R. Briefel, T. Manis, D. H. Gordon, A. D. Nicastri, and E. A. Friedman, "Recurrent renal vein thrombosis consequent to membranous glomerulonephritis," Clinical Nephrology, vol. 10, no. 1, pp. 32-37, 1978.

[29] R. D. Wagoner, A. W. Stanson, K. E. Holley, and C. S. Winter, "Renal vein thrombosis in idiopathic membranous glomerulopathy and nephrotic syndrome: incidence and significance," Kidney International, vol. 23, no. 2, pp. 368-374, 1983.

[30] T. H. Tran, G. J. Hughes, C. Greenfeld, and J. T. Pham, "Overview of current and alternative therapies for idiopathic membranous nephropathy," Pharmacotherapy, vol. 35, no. 4, pp. 396-411, 2015.

[31] H. Huh, H. Lee, J. P. Lee et al., "Factors affecting the longterm outcomes of idiopathic membranous nephropathy," BMC Nephrology, vol. 18, no. 1, article 104, 2017.

[32] C. C. Mok, "Membranous nephropathy in systemic lupus erythematosus: a therapeutic enigma," Nature Reviews Nephrology, vol. 5, no. 4, pp. 212-220, 2009.

[33] E. F. Rodriguez, S. H. Nasr, C. P. Larsen, S. Sethi, M. E. Fidler, and L. D. Cornell, "Membranous nephropathy with crescents: a series of 19 cases," American Journal of Kidney Diseases, vol. 64, no. 1, pp. 66-73, 2014.

[34] P. Ronco and H. Debiec, "Antigen identification in membranous nephropathy moves toward targeted monitoring and new therapy," Journal of the American Society of Nephrology, vol. 21, no. 4, pp. 564-569, 2010.

[35] Y. Yang, Y.-P. Ma, D.-P. Chen, L. Zhuo, and W.-G. Li, "A metaanalysis of antiviral therapy for hepatitis B virus-associated membranous nephropathy," PLoS ONE, vol. 11, no. 9, Article ID e0160437, 2016.

[36] Q. Weng, X. Li, H. Ren et al., "Membranous nephropathy associated with hepatitis $C$ virus infection treated with corticosteroids and Ledipasvir-Sofosbuvir: a case report and review of literature," Oncotarget, vol. 8, no. 13, pp. 22299-22303, 2017.
[37] B. Taton, K. Moreau, S. Lepreux et al., "Hepatitis E virus infection as a new probable cause of de novo membranous nephropathy after kidney transplantation," Transplant Infectious Disease, vol. 15, no. 6, pp. E211-E215, 2013.

[38] B. Thajudeen, S. G. John, N.-O. Ossai, I. B. Riaz, E. Bracamonte, and A. N. Sussman, "Membranous nephropathy with crescents in a patient with hashimoto's thyroiditis: a case report," Medicine, vol. 93, no. 8, article 98, 2014.

[39] K. N. Miyata, H. Kihira, M. Haneda, and Y. Nishio, "IgG4related tubulointerstitial nephritis associated with membranous nephropathy in two patients: remission after administering a combination of steroid and mizoribine," Case Reports in Nephrology, vol. 2014, pp. 1-6, 2014.

[40] J. A. Jefferson and W. G. Couser, “Therapy of membranous nephropathy associated with malignancy and secondary causes," Seminars in Nephrology, vol. 23, no. 4, pp. 400-405, 2003.

[41] D. Collister, C. Sathianathan, K. Ryz, M. Karpinski, K. Bernstein, and I. W. Gibson, "ANCA associated vasculitis secondary to levamisole-adultered cocaine with associated membranous nephropathy: a case series," American Journal of Nephrology, vol. 45, no. 3, pp. 209-216, 2017.

[42] R. P. S. Kumar, N. S. Prasad, S. Tirumavalavan, and M. E. Fernando, "D-penicillamine-induced membranous nephropathy," Indian Journal of Nephrology, vol. 24, no. 3, pp. 195-196, 2014.

[43] S. Manabe, M. Banno, M. Nakano et al., "Bucillamine-induced membranous nephropathy with crescent formation in a patient with rheumatoid arthritis: case report and literature review," Case Reports in Nephrology and Dialysis, vol. 5, no. 1, pp. 30-38, 2015.

[44] F. A. Nawaz, C. P. Larsen, and M. L. Troxell, "Membranous nephropathy and nonsteroidal anti-inflammatory agents," American Journal of Kidney Diseases, vol. 62, no. 5, pp. 1012-1017, 2013.

[45] M. Knehtl, H. Debiec, P. Kamgang et al., "A case of phospholipase $\mathrm{A} 2$ receptor-positive membranous nephropathy preceding sarcoid-associated granulomatous tubulointerstitial nephritis," American Journal of Kidney Diseases, vol. 57, no. 1, pp. 140-143, 2011.

[46] T. Stehlé, D. Joly, P. Vanhille et al., "Clinicopathological study of glomerular diseases associated with sarcoidosis: A multicenter study," Orphanet Journal of Rare Diseases, vol. 8, article 65, 2013.

[47] B. Svobodova, E. Honsova, P. Ronco, V. Tesar, and H. Debiec, "Kidney biopsy is a sensitive tool for retrospective diagnosis of PLA2R-related membranous nephropathy," Nephrology Dialysis Transplantation, vol. 28, no. 7, pp. 1839-1844, 2013.

[48] T. Stehlé, J.-J. Boffa, P. Lang, D. Desvaux, D. Sahali, and V. Audard, "Kidney involvement in sarcoidosis," La Revue de Médecine Interne, vol. 34, no. 9, pp. 538-544, 2013.

[49] T. Stehlé, V. Audard, P. Ronco, and H. Debiec, "Phospholipase A2 receptor and sarcoidosis-associated membranous nephropathy," Nephrology Dialysis Transplantation, vol. 30, no. 6, pp. 1047-1050, 2015.

[50] A. Gedalia, T. A. Khan, A. K. Shetty, V. R. Dimitriades, and L. R. Espinoza, "Childhood sarcoidosis: Louisiana experience," Clinical Rheumatology, vol. 35, no. 7, pp. 1879-1884, 2016.

[51] C. J. Byrne-Dugan, A. B. Collins, A. Q. Lam, and I. Batal, "Membranous nephropathy as a manifestation of graft-versus-host disease: Association with HLA antigen typing, phospholipase A2 receptor, and C4d," American Journal of Kidney Diseases, vol. 64, no. 6, pp. 987-993, 2014. 
[52] A. Abudayyeh, L. D. Truong, L. H. Beck, D. M. Weber, K. Rezvani, and M. Abdelrahim, "Membranous nephropathy in autologous hematopoietic stem cell transplant: Autologous graft-versus-host disease or autoimmunity induction?" Clinical Kidney Journal, vol. 8, no. 4, pp. 440-444, 2015.

[53] J. Zheng, Y. Xiao, Y. Yao et al., "Comparison of urinary biomarkers for early detection of acute kidney injury after cardiopulmonary bypass surgery in infants and young children," Pediatric Cardiology, vol. 34, no. 4, pp. 880-886, 2013.

[54] A. Omokawa, A. Komatsuda, M. Nara et al., "Distribution of glomerular IgG subclass deposits in patients with membranous nephropathy and anti-U1 ribonucleoprotein antibody.", Nephrology, dialysis, transplantation : official publication of the European Dialysis and Transplant Association - European Renal Association, vol. 27, no. 5, pp. 1937-1941, 2012.

[55] T. Doi, M. Mayumi, K. Kanatsu, F. Suehiro, and Y. Hamashima, "Distribution of IgG subclasses in membranous nephropathy," Clinical \& Experimental Immunology, vol. 58, no. 1, pp. 57-62, 1984.

[56] L.-H. Noël, P. Aucouturier, R. C. Monteiro, J.-L. Preud'homme, and P. Lesavre, "Glomerular and serum immunoglobulin G subclasses in membranous nephropathy and anti-glomerular basement membrane nephritis," Clinical Immunology and Immunopathology, vol. 46, no. 2, pp. 186-194, 1988.

[57] R. Hiramatsu, Y. Ubara, N. Sawa et al., "Clinicopathological analysis of allogeneic hematopoietic stem cell transplantationrelated membranous glomerulonephritis," Human Pathology, vol. 50, pp. 187-194, 2016.

[58] H. Ohtani, H. Wakui, A. Komatsuda et al., "Distribution of glomerular IgG subclass deposits in malignancy-associated membranous nephropathy," Nephrology Dialysis Transplantation, vol. 19, no. 3, pp. 574-579, 2004.

[59] Z. Qu, G. Liu, J. Li et al., "Absence of glomerular IgG4 deposition in patients with membranous nephropathy may indicate malignancy," Nephrology, Dialysis, Transplantation: Official Publication of The European Dialysis and Transplant Association-European Renal Association, vol. 27, no. 5, pp. 19311937, 2012.

[60] J. Lönnbro-Widgren, K. Ebefors, J. Mölne, J. Nyström, and B. Haraldsson, "Glomerular IgG subclasses in idiopathic and malignancy-associated membranous nephropathy," Clinical Kidney Journal, vol. 8, no. 4, pp. 433-439, 2015.

[61] H.-R. Dong, Y.-Y. Wang, X.-H. Cheng et al., "Retrospective study of phospholipase A2 receptor and IgG subclasses in glomerular deposits in Chinese patients with membranous nephropathy," PLoS ONE, vol. 11, no. 5, Article ID e0156263, 2016.

[62] S. Asmandar, M.-L. Figuères, J.-M. Goujon, L.-H. Noël, and A. Hummel, "Diagnostic value of IgG subtypes in membranous nephropathy: A case report," Néphrologie \& Thérapeutique, vol. 11, no. 3, article 780, pp. 169-172, 2015.

[63] A. Omokawa, A. Komatsuda, M. Hirokawa, and H. Wakui, "Membranous nephropathy with monoclonal IgG4 deposits and associated IgG4-related lung disease," Clinical Kidney Journal, vol. 7, no. 5, 2014.

[64] C. Lefaucheur, B. Stengel, and D. Nochy, "Membranous nephropathy and cancer: epidemiologic evidence and determinants of high-risk cancer association," Kidney International, vol. 70, no. 8, pp. 1510-1517, 2006.

[65] B. Alnasrallah, J. F. Collins, and L. J. Zwi, "Malignancy in Membranous Nephropathy: Evaluation of Incidence," International Journal of Nephrology, vol. 2017, Article ID 8409829, pp. 1-7, 2017.
[66] R. Hirschberg and S. Wang, "Proteinuria and growth factors in the development of tubulointerstitial injury and scarring in kidney disease," Current Opinion in Nephrology and Hypertension, vol. 14, no. 1, pp. 43-52, 2005.

[67] K. Zandi-Nejad, A. A. Eddy, R. J. Glassock, and B. M. Brenner, "Why is proteinuria an ominous biomarker of progressive kidney disease?" Kidney International Supplements, vol. 66, no. 92, pp. S76-S89, 2004.

[68] E. Hoxha, S. Harendza, H. Pinnschmidt, U. Panzer, and R. A. K. Stahl, "M-type phospholipase A2 receptor autoantibodies and renal function in patients with primary membranous nephropathy," Clinical Journal of the American Society of Nephrology, vol. 9, no. 11, pp. 1883-1890, 2014.

[69] T. Takano, H. Elimam, and A. V. Cybulsky, "Complementmediated cellular injury," Seminars in Nephrology, vol. 33, no. 6, pp. 586-601, 2013.

[70] F. C. Fervenza, G. Downer, L. H. Beck Jr., and S. Sethi, "IgG4-related tubulointerstitial nephritis with membranous nephropathy," American Journal of Kidney Diseases, vol. 58, no. 2, pp. 320-324, 2011.

[71] H. Ma, D. G. Sandor, and L. H. Beck, "The role of complement in membranous nephropathy," Seminars in Nephrology, vol. 33, no. 6, pp. 531-542, 2013.

[72] P. Ronco and H. Debiec, "Membranous nephropathy: a fairy tale for immunopathologists, nephrologists and patients," Molecular Immunology, vol. 68, no. 1, pp. 57-62, 2015.

[73] P. Ronco, H. Debiec, and V. Guigonis, "Mechanisms of Disease: Alloimmunization in renal diseases," Nature Clinical Practice Nephrology, vol. 2, no. 7, pp. 388-397, 2006.

[74] B. M. Coupes, S. P. Kon, P. E. C. Brenchley, C. D. Short, and N. P. Mallick, "The temporal relationship between urinary c5b9 and c3dg and clinical parameters in human membranous nephropathy," Nephrology Dialysis Transplantation, vol. 8, no. 5, pp. 397-401, 1993.

[75] A. A. Papagianni, E. Alexopoulos, M. Leontsini, and M. Papadimitriou, "C5b-9 and adhesion molecules in human idiopathic membranous nephropathy," Nephrology Dialysis Transplantation, vol. 17, no. 1, pp. 57-63, 2002.

[76] W. J. Liu, Z. Li, X. Chen et al., "Blockage of the lysosomedependent autophagic pathway contributes to complement membrane attack complex-induced podocyte injury in idiopathic membranous nephropathy," Scientific Reports, vol. 7, article 8643, 2017.

[77] M. Beeken, M. T. Lindenmeyer, S. M. Blattner et al., "Alterations in the ubiquitin proteasome system in persistent but not reversible proteinuric diseases," Journal of the American Society of Nephrology, vol. 25, no. 11, pp. 2511-2525, 2014.

[78] A. V. Cybulsky, "The intersecting roles of endoplasmic reticulum stress, ubiquitin-proteasome system, and autophagy in the pathogenesis of proteinuric kidney disease," Kidney International, vol. 84, no. 1, pp. 25-33, 2013.

[79] C. Meyer-Schwesinger, T. N. Meyer, H. Sievert et al., "Ubiquitin C-terminal hydrolase-L1 activity induces polyubiquitin accumulation in podocytes and increases proteinuria in rat membranous nephropathy," The American Journal of Pathology, vol. 178, no. 5, pp. 2044-2057, 2011.

[80] L. Biancone, S. David, V. Della Pietra, G. Montrucchio, V. Cambi, and G. Camussi, "Alternative pathway activation of complement by cultured human proximal tubular epithelial cells," Kidney International, vol. 45, no. 2, pp. 451-460, 1994.

[81] G. Camussi, M. Rotunno, G. Segoloni, J. R. Brentjens, and G. A. Andres, "In vitro alternative pathway activation of complement 
by the brush border of proximal tubules of normal rat kidney," The Journal of Immunology, vol. 128, no. 4, pp. 1659-1663, 1982.

[82] A. Zaferani, R. R. Vivès, P. Van Der Pol et al., "Identification of tubular heparan sulfate as a docking platform for the alternative complement component properdin in proteinuric renal disease," The Journal of Biological Chemistry, vol. 286, no. 7, pp. 5359-5367, 2011.

[83] M. Harboe, C. Johnson, S. Nymo et al., "Properdin binding to complement activating surfaces depends on initial C3b deposition," Proceedings of the National Acadamy of Sciences of the United States of America, vol. 114, no. 4, pp. E534-E539, 2017.

[84] H. Gaarkeuken, M. A. Siezenga, K. Zuidwijk et al., "Complement activation by tubular cells is mediated by properdin binding," American Journal of Physiology-Renal Physiology, vol. 295, no. 5, pp. F1397-F1403, 2008.

[85] P. Ronco and H. Debiec, "Molecular pathomechanisms of membranous nephropathy: From heymann nephritis to alloimmunization," Journal of the American Society of Nephrology, vol. 16, no. 5, pp. 1205-1213, 2005.

[86] N. M. Tomas, E. Hoxha, A. T. Reinicke et al., "Autoantibodies against thrombospondin type 1 domain-containing $7 \mathrm{~A}$ induce membranous nephropathy," The Journal of Clinical Investigation, vol. 126, no. 7, pp. 2519-2532, 2016.

[87] C.-Y. Chen, G. Salles, M. F. Seldin, A. E. Kister, E. L. Reinherz, and M. A. Shipp, "Murine common acute lymphoblastic leukemia antigen (CD10 neutral endopeptidase 24.11): Molecular characterization, chromosomal localization, and modeling of the active site," The Journal of Immunology, vol. 148, no. 9, pp. 2817-2825, 1992.

[88] P. Hu, Q. Xuan, B. Hu, L. Lu, and Y. H. Qin, "Anti-neutral endopeptidase, natriuretic peptides disarrangement, and proteinuria onset in membranous nephropathy," Molecular Biology Reports, vol. 40, no. 4, pp. 2963-2967, 2013.

[89] J. L. Nortier, H. Debiec, Y. Tournay et al., "Neonatal disease in neutral endopeptidase alloimmunization: Lessons for immunological monitoring," Pediatric Nephrology, vol. 21, no. 10, pp. 1399-1405, 2006.

[90] H. Debiec, J. Nauta, F. Coulet et al., "Role of truncating mutations in MME gene in fetomaternal alloimmunisation and antenatal glomerulopathies," The Lancet, vol. 364, no. 9441, pp. 1252-1259, 2004.

[91] H. Debiec, V. Guigonis, B. Mougenot et al., "Extra-membranous glomerulonephritis: report of a family and new physiopathological concepts," Bulletin de Lacademie Nationale De Medecine, vol. 187, no. 5, pp. 921-932, 2003.

[92] M. Vivarelli, F. Emma, T. Pellé et al., "Genetic homogeneity but IgG subclass-dependent clinical variability of alloimmune membranous nephropathy with anti-neutral endopeptidase antibodies," Kidney International, vol. 87, no. 3, pp. 602-609, 2015.

[93] C. Murtas, L. Allegri, and G. M. Ghiggeri, "Circulating antipodocyte antibodies in membranous nephropathy: New findings," American Journal of Kidney Diseases, vol. 62, no. 1, pp. 12-15, 2013.

[94] R. Ramachandran, V. Kumar, A. Kumar et al., "PLA2R antibodies, glomerular PLA2R deposits and variations in PLA2R1 and HLA-DQA1 genes in primary membranous nephropathy in South Asians," Nephrology Dialysis Transplantation, vol. 31, no. 9, pp. 1486-1493, 2016.

[95] G. Gupta, H. Fattah, R. Ayalon et al., "Pre-transplant phospholipase $\mathrm{A} 2$ receptor autoantibody concentration is associated with clinically significant recurrence of membranous nephropathy post-kidney transplantation," Clinical Transplantation, vol. 30, no. 4, pp. 461-469, 2016.

[96] A. Behnert, M. J. Fritzler, B. Teng et al., "An anti-phospholipase $\mathrm{A}_{2}$ receptor quantitative immunoassay and epitope analysis in membranous nephropathy reveals different antigenic domains of the receptor," PLoS ONE, vol. 8, no. 4, Article ID e61669, 2013.

[97] G. Sugahara, J. Kamiie, R. Kobayashi, T. Mineshige, and K. Shirota, "Expression of phospholipase A2 receptor in primary cultured podocytes derived from dog kidneys," Journal of Veterinary Medical Science, vol. 78, no. 5, pp. 895-899, 2016.

[98] H. Debiec, M. Hanoy, A. Francois et al., "Recurrent membranous nephropathy in an allograft caused by IgG3 $\kappa$ targeting the PLA2 receptor," Journal of the American Society of Nephrology, vol. 23, no. 12, pp. 1949-1954, 2012.

[99] P. T. Klouda, E. J. Acheson, F. S. Goldby et al., "Strong association between idiopathic membranous nephropathy and HLA-DRW3," The Lancet, vol. 314, no. 8146, pp. 770-771, 1979.

[100] S. Mezzano, G. Rojas, L. Ardiles et al., "Idiopathic membranous nephropathy, associated with HLA-DRw3 and not related to monocyte-phagocyte system Fc receptor dysfunction, in father and son," Nephron, vol. 58, no. 3, pp. 320-324, 1991.

[101] H. C. Stanescu, M. Arcos-Burgos, A. Medlar et al., "Risk HLA-DQA1 and $\mathrm{PLA}_{2} \mathrm{R} 1$ alleles in idiopathic membranous nephropathy," The New England Journal of Medicine, vol. 364, no. 7, pp. 616-626, 2011.

[102] S. Kim, H. J. Chin, K. Y. Na et al., "Single nucleotide polymorphisms in the phospholipase $\mathrm{A}_{2}$ receptor gene are associated with genetic susceptibility to idiopathic membranous nephropathy," Nephron Clinical Practice, vol. 117, no. 3, pp. c253c258, 2011.

[103] G. Bullich, J. Ballarín, A. Oliver et al., "HLA-DQA1 and PLA2R1 polymorphisms and risk of idiopathic membranous nephropathy," Clinical Journal of the American Society of Nephrology, vol. 9, no. 2, pp. 335-343, 2014.

[104] Y.-H. Liu, C.-H. Chen, S.-Y. Chen et al., "Association of phospholipase A2 receptor 1 polymorphisms with idiopathic membranous nephropathy in Chinese patients in Taiwan," Journal of Biomedical Science, vol. 17, article 81, 2010.

[105] P. Ruggenenti, H. Debiec, B. Ruggiero et al., "Anti-Phospholipase A2 receptor antibody titer predicts post-rituximab outcome of membranous nephropathy," Journal of the American Society of Nephrology, vol. 26, no. 10, pp. 2545-2558, 2015.

[106] M. Fresquet, T. A. Jowitt, and J. Gummadova, "Identification of a major epitope recognized by PLA2R autoantibodies in primary membranous nephropathy," Journal of the American Society of Nephrology, vol. 26, no. 2, pp. 302-313, 2015.

[107] L. Kao, V. Lam, M. Waldman, R. J. Glassock, and Q. Zhu, "Identification of the immunodominant epitope region in phospholipase A2 receptor-mediating autoantibody binding in idiopathic membranous nephropathy," Journal of the American Society of Nephrology, vol. 26, no. 2, pp. 291-301, 2015.

[108] B. Seitz-Polski, G. Dolla, C. Payre et al., "Epitope spreading of autoantibody response to PLA2R associates with poor prognosis in membranous nephropathy," Journal of the American Society of Nephrology, vol. 27, no. 5, pp. 1517-1533, 2016.

[109] B. Seitz-Polski, G. Lambeau, and V. Esnault, "Membranous nephropathy: Pathophysiology and natural history," Nephrologie \& Therapeutique, vol. 13, supplement 1, pp. S75-S81, 2017.

[110] A. Pozdzik, I. Beukinga, C. Gu-Trantien, K. Willard-Gallo, J. Nortier, and O. Pradier, "Circulating (CD3-CD19+CD20IgD-CD $27^{\text {high }} \mathrm{CD} 38^{\text {high }}$ ) plasmablasts: a promising cellular 
biomarker for immune activity for anti-PLA2R1 related membranous nephropathy?" Mediators of Inflammation, vol. 2016, Article ID 7651024, pp. 1-10, 2016.

[111] M. Rosenzwajg, E. Languille, H. Debiec et al., "B- and T-cell subpopulations in patients with severe idiopathic membranous nephropathy may predict an early response to rituximab," Kidney International, vol. 92, no. 1, pp. 227-237, 2016.

[112] D. Roccatello, S. Sciascia, D. Di Simone et al., "New insights into immune mechanisms underlying response to Rituximab in patients with membranous nephropathy: a prospective study and a review of the literature," Autoimmunity Reviews, vol. 15, no. 6, pp. 529-538, 2016.

[113] A. Barbari, "Pre- and posttransplant refractory idiopathic membranous glomerulonephritis: the forgotten potential culprit," Experimental and Clinical Transplantation, vol. 15, no. 5, pp. 483-489, 2017.

[114] L. Lin, W. M. Wang, X. X. Pan et al., "Biomarkers to detect membranous nephropathy in Chinese patients," Oncotarget, vol. 7, no. 42, pp. 67868-67879, 2016.

[115] E. Hoxha, S. Harendza, H. O. Pinnschmidt et al., "Spontaneous remission of proteinuria is a frequent event in phospholipase A2 receptor antibody-negative patients with membranous nephropathy," Nephrology Dialysis Transplantation, vol. 30, no. 11, pp. 1862-1869, 2015.

[116] J. Wang, Z. Cui, J. Lu et al., "Circulating Antibodies against Thrombospondin Type-I Domain-Containing 7A in Chinese Patients with Idiopathic Membranous Nephropathy,' Clinical Journal of the American Society of Nephrology, vol. 12, no. 10, pp. 1642-1651, 2017.

[117] E. Hoxha, T. Wiech, P. R. Stahl et al., "A mechanism for cancer-associated membranous nephropathy," The New England Journal of Medicine, vol. 374, no. 20, pp. 1995-1996, 2016.

[118] P. R. Stahl, E. Hoxha, T. Wiech, C. Schröder, R. Simon, and R. A. K. Stahl, "THSD7A expression in human cancer," Genes, Chromosomes and Cancer, vol. 56, no. 4, pp. 314-327, 2017.

[119] N. D. Quach, J. N. Mock, N. E. Scholpa et al., "Role of the phospholipase A2 receptor in liposome drug delivery in prostate cancer cells," Molecular Pharmaceutics, vol. 11, no. 10, pp. 3443-3451, 2014.

[120] J.-G. Lee, S.-H. Lee, D.-W. Park et al., "Toll-like receptor 9stimulated monocyte chemoattractant protein-1 is mediated via JNK-cytosolic phospholipase A2-ROS signaling," Cellular Signalling, vol. 20, no. 1, pp. 105-111, 2008.

[121] E. Chiricozzi, S. Fernandez-Fernandez, V. Nardicchi, A. Almeida, J. P. Bolaños, and G. Goracci, "Group IIA secretory phospholipase A2 (GIIA) mediates apoptotic death during NMDA receptor activation in rat primary cortical neurons," Journal of Neurochemistry, vol. 112, no. 6, pp. 1574-1583, 2010.

[122] Y. Pan, J. Wan, Y. Liu et al., "sPLA2 IB induces human podocyte apoptosis via the M-type phospholipase A2 receptor," Scientific Reports, vol. 4, article no. 6660, 2014.

[123] J. E. Murphy-Ullrich and E. H. Sage, "Revisiting the matricellular concept," Matrix Biology, vol. 37, pp. 1-14, 2014.

[124] C. Meyer-Schwesinger, G. Lambeau, and R. A. K. Stahl, “The authors reply: Thrombospondin type-1 domain-containing 7A in idiopathic membranous nephropathy," The New England Journal of Medicine, vol. 372, no. 11, pp. 1074-1075, 2015.

[125] M. Gödel, F. Grahammer, and T. B. Huber, "Thrombospondin type-1 domain-containing 7A in idiopathic membranous nephropathy," The New England Journal of Medicine, vol. 372, no. 11, article 1073, 2015.
[126] N. M. Tomas, C. Meyer-Schwesinger, H. von Spiegel et al., "A heterologous model of thrombospondin type 1 domaincontaining 7A-associated membranous nephropathy," Journal of the American Society of Nephrology, vol. 28, no. 10, 2017.

[127] H. Debiec and P. Ronco, "PLA2R autoantibodies and PLA2R glomerular deposits in membranous nephropathy," The New England Journal of Medicine, vol. 364, no. 7, pp. 689-690, 2011.

[128] H. Debiec, L. Martin, C. Jouanneau et al., "Autoantibodies specific for the phospholipase A 2 receptor in recurrent and de novo membranous nephropathy," American Journal of Transplantation, vol. 11, no. 10, pp. 2144-2152, 2011.

[129] F. Pourcine, K. Dahan, F. Mihout et al., "Prognostic value of PLA2R autoimmunity detected by measurement of antiPLA2R antibodies combined with detection of PLA2R antigen in membranous nephropathy: A single-centre study over 14 years," PLoS ONE, vol. 12, no. 3, Article ID e0173201, 2017.

[130] E. Hoxha, U. Kneiler, G. Stege et al., "Enhanced expression of the M-type phospholipase A2 receptor in glomeruli correlates with serum receptor antibodies in primary membranous nephropathy," Kidney International, vol. 82, no. 7, pp. 797-804, 2012.

[131] W. Qin, L. H. Beck Jr., C. Zeng et al., "Anti-phospholipase A2 receptor antibody in membranous nephropathy," Journal of the American Society of Nephrology, vol. 22, no. 6, pp. 1137-1143, 2011.

[132] K. Hihara, M. Iyoda, S. Tachibana et al., "Anti-phospholipase A2 receptor (PLA2R) antibody and glomerular PLA2R expression in Japanese patients with membranous nephropathy," PLoS ONE, vol. 11, no. 6, Article ID e0158154, 2016.

[133] R. Hu, G. Xing, H. Wu, and Z. Zhang, "Clinicopathological features of idiopathic membranous nephropathy combined with IgA nephropathy: A retrospective analysis of 9 cases," Diagnostic Pathology, vol. 11, no. 1, article 86, 2016.

[134] Q. Xie, Y. Li, J. Xue et al., "Renal Phospholipase A2 Receptor in Hepatitis B Virus-Associated Membranous Nephropathy," American Journal of Nephrology, vol. 41, no. 4-5, pp. 345-353, 2015.

[135] L. H. Beck Jr., F. C. Fervenza, D. M. Beck et al., "Rituximabinduced depletion of anti-PLA2R autoantibodies predicts response in membranous nephropathy," Journal of the American Society of Nephrology, vol. 22, no. 8, pp. 1543-1550, 2011.

[136] J. Wang, Q. Xie, Z. Sun et al., "Response to immunosuppressive therapy in PLA2R- associated and non-PLA2R- associated idiopathic membranous nephropathy: a retrospective, multicenter cohort study," BMC Nephrology, vol. 18, no. 1, article 227, 2017.

[137] E. Hoxha, I. Thiele, G. Zahner, U. Panzer, S. Harendza, and R. A. K. Stahl, "Phospholipase A2 receptor autoantibodies and clinical outcome in patients with primary membranous nephropathy," Journal of the American Society of Nephrology, vol. 25, no. 6, pp. 1357-1366, 2014.

[138] C. P. Larsen and P. D. Walker, "Phospholipase A2 receptor (PLA2R) staining is useful in the determination of de novo versus recurrent membranous glomerulopathy," Transplantation, vol. 95, no. 10, pp. 1259-1262, 2013.

[139] H. Saito, Y. Hamasaki, A. Tojo, Y. Shintani, A. Shimizu, and M. Nangaku, "Phospholipase A2 receptor positive membranous nephropathy long after living donor kidney transplantation between identical twins," Nephrology, vol. 20, no. 2, pp. 101-104, 2015.

[140] A. Grupper, L. D. Cornell, F. C. Fervenza, L. H. Beck, E. Lorenz, and F. G. Cosio, "Recurrent Membranous Nephropathy after Kidney Transplantation: Treatment and Long-Term Implications," Transplantation, vol. 100, no. 12, pp. 2710-2716, 2016. 
[141] C. D. Blosser, R. Ayalon, R. Nair, C. Thomas, and L. H. Beck Jr., "Very early recurrence of anti-phospholipase A 2 receptor-positive membranous nephropathy after transplantation," American Journal of Transplantation, vol. 12, no. 6, pp. 1637-1642, 2012.

[142] H. Wakui, H. Imai, A. Komatsuda, and A. B. Miura, "Circulating antibodies against $\alpha$-enolase in patients with primary membranous nephropathy (MN)," Clinical \& Experimental Immunology, vol. 118, no. 3, pp. 445-450, 1999.

[143] Y. Kimura, N. Miura, H. Debiec et al., "Circulating antibodies to $\alpha$-enolase and phospholipase A2 receptor and composition of glomerular deposits in Japanese patients with primary or secondary membranous nephropathy," Clinical and Experimental Nephrology, vol. 21, no. 1, pp. 117-126, 2017.

[144] M. Prunotto, M. L. Carnevali, G. Candiano et al., "Autoimmunity in membranous nephropathy targets aldose reductase and SOD2," Journal of the American Society of Nephrology, vol. 21, no. 3, pp. 507-519, 2010.

[145] M. Gödel and T. B. Huber, "Immunosuppression for membranous nephropathy," The Lancet, vol. 381, no. 9884, article 2161, 2013.

[146] J. M. Hofstra, F. C. Fervenza, and J. F. M. Wetzels, “Treatment of idiopathic membranous nephropathy," Nature Reviews Nephrology, vol. 9, no. 8, pp. 443-458, 2013.

[147] C. Ponticelli and R. J. Glassock, "Glomerular diseases: membranous nephropathy-a modern view," Clinical Journal of the American Society of Nephrology, vol. 9, no. 3, pp. 609-616, 2014.

[148] J. Radhakrishnan and D. C. Cattran, "The KDIGO practice guideline on glomerulonephritis: Reading between the (guide)lines-application to the individual patient," Kidney International, vol. 82, no. 8, pp. 840-856, 2012.

[149] H. Shiiki, T. Saito, Y. Nishitani et al., "Prognosis and risk factors for idiopathic membranous nephropathy with nephrotic syndrome in Japan," Kidney International, vol. 65, no. 4, pp. 1400-1407, 2004.

[150] R. Dikow, P. Quentmeier, V. Schwenger et al., "Optimal blood pressure control versus additional immunosuppressive therapy in idiopathic membranous nephropathy - A retrospective analysis," Clinical Nephrology, vol. 72, no. 5, pp. 366-372, 2009.

[151] J. Xu, X. Hu, J. Xie, and N. Chen, "Management of Membranous Nephropathy in Asia," Kidney Diseases, vol. 1, no. 2, pp. 119-125, 2015.

[152] T. Alfaadhel and D. Cattran, "Management of Membranous Nephropathy in Western Countries," Kidney Diseases, vol. 1, no. 2, pp. 126-137, 2015.

[153] D. C. Cattran, "Management of membranous nephropathy," Minerva Urologica e Nefrologica, vol. 54, no. 1, pp. 19-27, 2002.

[154] J. A. J. G. van den Brand, P. R. Van Dijk, J. M. Hofstra, and J. F. M. Wetzels, "Long-term outcomes in idiopathic membranous nephropathy using a restrictive treatment strategy," Journal of the American Society of Nephrology, vol. 25, no. 1, pp. 150-158, 2014.

[155] D. A. Kanigicherla, C. D. Short, S. A. Roberts et al., "Long-term outcomes of persistent disease and relapse in primary membranous nephropathy," Nephrology Dialysis Transplantation, vol. 31, no. 12, pp. 2108-2114, 2016.

[156] Y. Chen, A. Schieppati, X. Chen et al., "Immunosuppressive treatment for idiopathic membranous nephropathy in adults with nephrotic syndrome," Cochrane Database of Systematic Reviews, vol. 10, article CD004293, 2014.

[157] M. Praga, “Tacrolimus in membranous nephropathy," Kidney International, vol. 74, no. 6, article 824, 2008.
[158] M. Praga, V. Barrio, G. F. Juárez, and J. Luño, “Tacrolimus monotherapy in membranous nephropathy: A randomized controlled trial," Kidney International, vol. 71, no. 9, pp. 924930, 2007.

[159] R. Ramachandran, H. K. Hn, V. Kumar et al., "Tacrolimus combined with corticosteroids versus Modified Ponticelli regimen in treatment of idiopathic membranous nephropathy: Randomized control trial," Nephrology, vol. 21, no. 2, pp. 139146, 2016.

[160] S. Ren, Y. Wang, L. Xian et al., "Comparative effectiveness and tolerance of immunosuppressive treatments for idiopathic membranous nephropathy: A network meta-analysis," PLoS ONE, vol. 12, no. 9, p. e0184398, 2017.

[161] X. Yu, L. Ruan, Z. Qu et al., "Low-dose cyclosporine in treatment of membranous nephropathy with nephrotic syndrome: effectiveness and renal safety," Renal Failure, vol. 39, no. 1, pp. 688-697, 2017.

[162] G. Xie, J. Xu, C. Ye et al., "Immunosuppressive Treatment for Nephrotic Idiopathic Membranous Nephropathy: A MetaAnalysis Based on Chinese Adults," PLoS ONE, vol. 7, no. 9, Article ID e44330, 2012.

[163] A. J. Branten, P. W. du Buf-Vereijken, M. Vervloet, and J. F. Wetzels, "Mycophenolate Mofetil in Idiopathic Membranous Nephropathy: A Clinical Trial With Comparison to a Historic Control Group Treated With Cyclophosphamide," American Journal of Kidney Diseases, vol. 50, no. 2, pp. 248-256, 2007.

[164] C. Ponticelli, "What is the role of rituximab in idiopathic membranous nephropathy?" Expert Review of Clinical Immunology, vol. 9, no. 1, pp. 13-16, 2013.

[165] M. A. Hladunewich, D. Cattran, L. H. Beck et al., "A pilot study to determine the dose and effectiveness of adrenocorticotrophic hormone (H.P. Acthar ${ }^{\circledR}$ Gel) in nephrotic syndrome due to idiopathic membranous nephropathy," Nephrology Dialysis Transplantation, vol. 29, no. 8, pp. 1570-1577, 2014.

[166] R. Stahl, E. Hoxha, and K. Fechner, "PLA2R autoantibodies and recurrent membranous nephropathy after transplantation," The New England Journal of Medicine, vol. 363, no. 5, pp. 496-498, 2010.

[167] P. Cravedi, G. Remuzzi, and P. Ruggenenti, "Rituximab in primary membranous nephropathy: first-line therapy, why not?" Nephron Clinical Practice, vol. 128, no. 3-4, pp. 261-269, 2014.

[168] K. Dahan, H. Debiec, E. Plaisier et al., "Rituximab for severe membranous nephropathy: a 6-month trial with extended follow-up," Journal of the American Society of Nephrology, vol. 28, no. 1, pp. 348-358, 2017.

[169] M. Fiorentino, F. Tondolo, F. Bruno et al., "Treatment with rituximab in idiopathic membranous nephropathy," Clinical Kidney Journal, vol. 9, no. 6, pp. 788-793, 2016.

[170] F. B. Cortazar, D. E. Leaf, C. T. Owens, K. Laliberte, W. F. Pendergraft, and J. L. Niles, "Combination therapy with rituximab, low-dose cyclophosphamide, and prednisone for idiopathic membranous nephropathy: a case series," BMC Nephrology, vol. 18, no. 1, pp. 1-10, 2017.

[171] J. A. van den Brand, P. Ruggenenti, A. Chianca et al., "Safety of Rituximab Compared with Steroids and Cyclophosphamide for Idiopathic Membranous Nephropathy," Journal of the American Society of Nephrology, vol. 28, no. 9, pp. 2729-2737, 2017.

[172] T. Katsuno, T. Ozaki, H. Kim et al., "Single-dose Rituximab Therapy for Refractory Idiopathic Membranous Nephropathy: A Single-center Experience," Internal Medicine, vol. 56, no. 13, pp. 1679-1686, 2017. 
[173] F. C. Fervenza, P. A. Canetta, S. J. Barbour et al., "A multicenter randomized controlled trial of rituximab versus cyclosporine in the treatment of idiopathic membranous nephropathy (mentor)," Nephron, vol. 130, no. 3, pp. 159-168, 2015.

[174] P. Cravedi, "Rituximab in Membranous Nephropathy: Not All Studies Are Created Equal," Nephron, vol. 135, no. 1, pp. 46-50, 2017.

[175] W. Cheungpasitporn, S. L. Kopecky, U. Specks, K. Bharucha, and F. C. Fervenza, "Non-ischemic cardiomyopathy after rituximab treatment for membranous nephropathy," Journal of Renal Injury Prevention, vol. 6, no. 1, pp. 18-25, 2017.

[176] R. Radhakrishnan, G. Basu, R. George, H. Parmar, and V. Tamilarasi, "Rituximab-induced urticarial dermatitis during the treatment of membranous nephropathy," Saudi Journal of Kidney Diseases and Transplantation, vol. 28, no. 3, pp. 657-660, 2017.

[177] A. A. Rigler, A. Jerman, A. Orsag et al., "Rituximab for the treatment of membranous nephropathy: a single-center experience," Clinical Nephrology, vol. 88, no. 13, pp. 27-31, 2017.

[178] G. Moroni, F. Depetri, L. Del Vecchio et al., "Low-dose rituximab is poorly effective in patients with primary membranous nephropathy," Nephrology, Dialysis, Transplantation: Official Publication of The European Dialysis and Transplant Association - European Renal Association, vol. 32, no. 10, pp. 1691-1696, 2017.

[179] M. Waldman, L. H. Beck, M. Braun, K. Wilkins, J. E. Balow, and H. A. Austin, "Membranous Nephropathy: Pilot Study of a Novel Regimen Combining Cyclosporine and Rituximab," Kidney International Reports, vol. 1, no. 2, pp. 73-84, 2016.

[180] A. Barbari, R. Chehadi, H. Kfoury Assouf et al., "Bortezomib as a Novel Approach to Early Recurrent Membranous Glomerulonephritis After Kidney Transplant Refractory to Combined Conventional Rituximab Therapy," Experimental And Clinical Transplantation: Official Journal of The Middle East Society for Organ Transplantation, vol. 15, no. 3, pp. 350-354, 2017.

[181] G. La Manna, O. Baraldi, V. Cunia et al., "Membranous glomerulonephritis (MGN), ongoing studies," Giornale Italiano Di Nefrologia, vol. 34, no. 5, pp. 113-118, 2017.

[182] P. Ruggenenti, F. C. Fervenza, and G. Remuzzi, "Treatment of membranous nephropathy: time for a paradigm shift," Nature Reviews Nephrology, vol. 13, no. 9, pp. 563-579, 2017.

[183] J.-F. Cambier and P. Ronco, "Onco-nephrology: glomerular diseases with cancer," Clinical journal of the American Society of Nephrology, vol. 7, no. 10, pp. 1701-1712, 2012.

[184] A. S. De Vriese, R. J. Glassock, K. A. Nath, S. Sethi, and F. C. Fervenza, "A Proposal for a serology-based approach to membranous nephropathy," Journal of the American Society of Nephrology, vol. 28, no. 2, pp. 421-430, 2017.

[185] W. G. Couser, "Primary Membranous Nephropathy," Clinical Journal of the American Society of Nephrology, vol. 12, no. 6, pp. 983-997, 2017. 


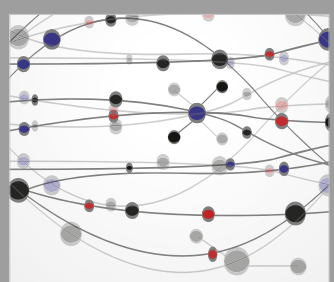

The Scientific World Journal
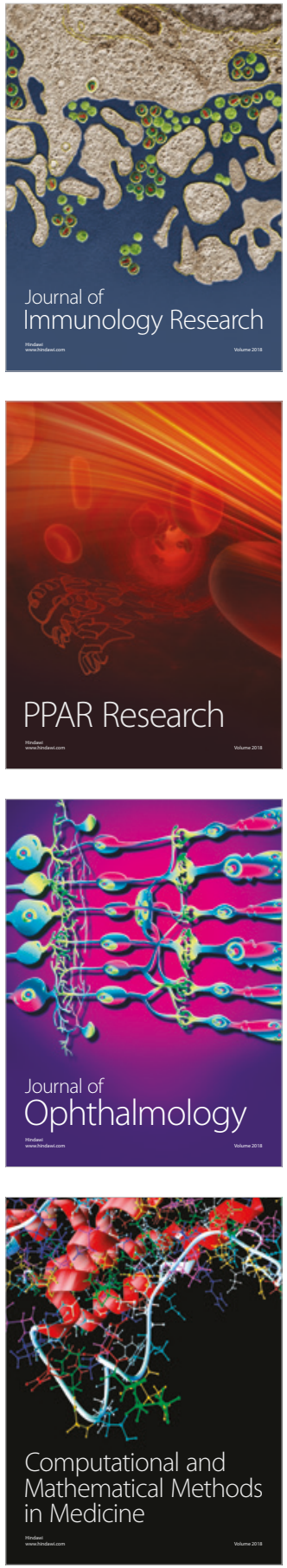

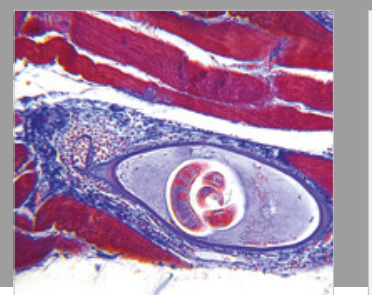

Gastroenterology Research and Practice

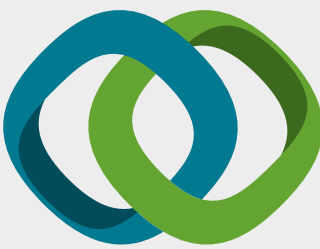

\section{Hindawi}

Submit your manuscripts at

www.hindawi.com
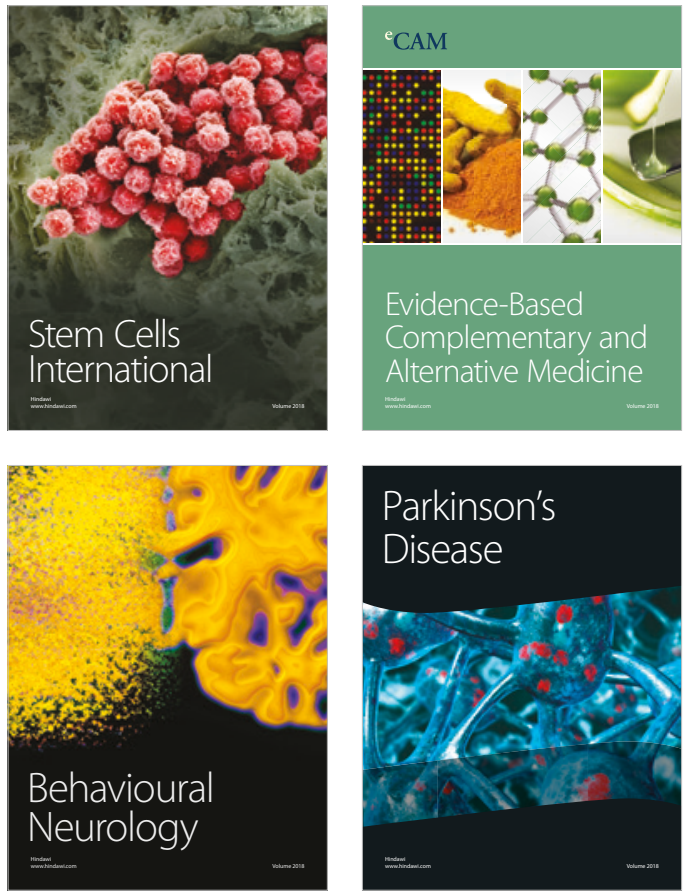

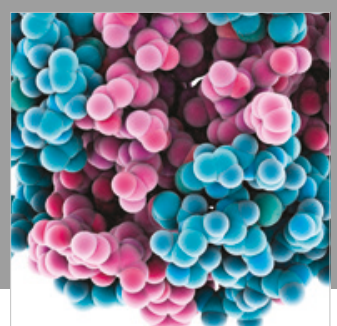

ournal of

Diabetes Research

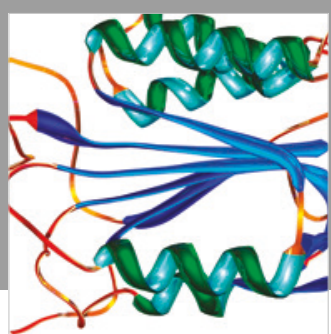

Disease Markers
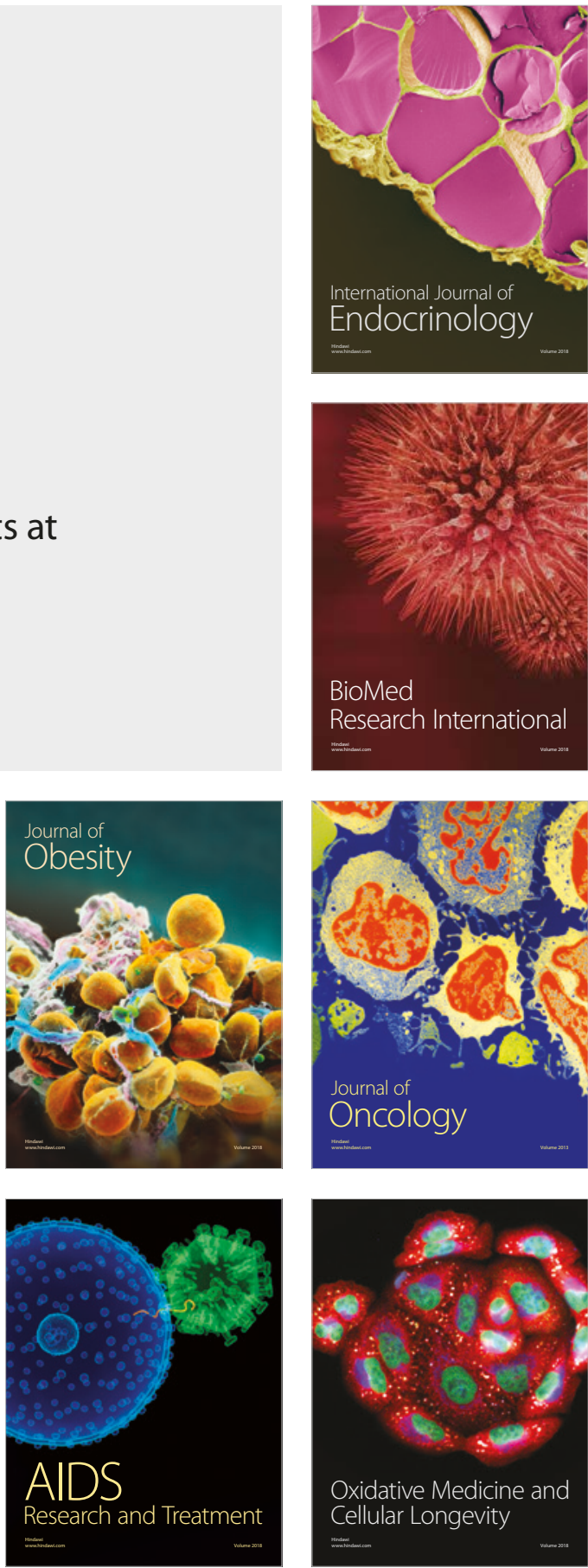